\title{
SETS OF UNIFORMLY ABSOLUTELY CONTINUOUS NORM IN SYMMETRIC SPACES OF MEASURABLE OPERATORS
}

\author{
P. G. DODDS, B. DE PAGTER, AND F. SUKOCHEV
}

\begin{abstract}
We characterise sets of uniformly absolutely continuous norm in strongly symmetric spaces of $\tau$-measurable operators. Applications are given to the study of relatively weakly compact and relatively compact sets and to compactness properties of operators dominated in the sense of complete positivity by compact or by Dunford-Pettis operators.
\end{abstract}

\section{INTRODUCTION}

This paper finds much of its inspiration from the classical characterisation of Dunford and Pettis of relatively weakly compact sets in $L_{1}$-spaces. In particular, a bounded subset $A$ of any abstract $L$-space $E$ is relatively weakly compact if and only if each disjoint sequence in its solid hull converges in norm to zero. In turn, each of these statements is equivalent to the assertion that $A$ is $E$-equicontinuous, or of uniformly absolutely continuous norm, in the sense that each downwards directed system in the dual of $E$ with infimum 0 should converge uniformly to 0 on the set $A$. Moreover, this latter statement is equivalent to the further statement that $A$ should be approximately order bounded in $E$, in the sense of Banach lattices. For further details, the reader is referred to Chapter 2 of the monograph [31] of Meyer-Nieberg.

The classical Dunford-Pettis theorem found non-trivial generalisations in several directions. In the setting of Banach lattices, Meyer-Nieberg 31] introduced the notion of an $L$-weakly compact set in a Banach lattice $E$, being a bounded subset of $E$ with the property that each disjoint sequence in its solid hull should be norm convergent to zero. He showed that, in a Banach lattice with order continuous norm, the class of $L$-weakly compact sets coincides with the class of approximately order bounded sets and that each $L$-weakly compact set was weakly compact, though the converse is not true in general. In a very different direction, the Dunford-Pettis theorem was extended by C.A. Akemann 2] (see 42]) to the predual $\mathcal{M}_{*}$ of an arbitrary von Neumann algebra. In particular, he showed that a bounded subset $K$ of the predual $\mathcal{M}_{*}$ is relatively weakly compact if and only if $K$ is of uniformly absolutely continuous norm in the sense that whenever $\left\{e_{\alpha}\right\}_{\alpha \in \mathcal{A}}$ is a decreasing net of projections in $\mathcal{M}$ with infimum zero, then $\lim _{\alpha}\left\|e_{\alpha} \phi e_{\alpha}\right\|=0$ uniformly on

Received by the editors July 25, 2013 and, in revised form, April 29, 2014.

2010 Mathematics Subject Classification. Primary 46L52; Secondary 46E30, 47A30.

Key words and phrases. Measurable operators, uniformly absolutely continuous norm, strongly symmetric spaces.

This work was partially supported by the Australian Research Council. 
$K$. However, there are substantial differences between the commutative and noncommutative settings; for example, it is no longer, in general, the case in the predual of a von Neumann algebra that the solid hull of a relatively weakly compact set is again relatively weakly compact.

A principal aim of this paper is to study subsets of uniformly absolutely continuous norm (in the above sense of Akemann) and their relation to weak compactness in the setting of strongly symmetric spaces of $\tau$-measurable operators affiliated with a semifinite von Neumann algebra $(\mathcal{M}, \tau)$. One of the key technical results (Theorem 3.12) which links each of the successive sections of this paper shows that, in strongly symmetric spaces with order continuous norm, a bounded set is of uniformly absolutely continuous norm if and only if it is approximately order bounded in a natural sense. In the case of the Haagerup $L_{p}$-spaces, such a characterisation has been given by Raynaud and $\mathrm{Xu}[38$ ] and in the special case of the predual of a non-atomic finite von Neumann algebra may be found in [26]. However, the methods of [38 are based on ultraproduct techniques, and it is not in general the case that the ultraproduct of an arbitrary family of semifinite von Neumann algebras is again semifinite. By way of contrast, our approach is more direct and is an extension of that of [26].

Applications to the study of relatively weakly compact sets are given in Section 4 and to relatively compact sets in Section 5 . While it is the case that a bounded set $\mathcal{A}$ of uniformly absolutely continuous norm in non-commutative symmetric spaces with order continuous norm is relatively weakly compact (Proposition 4.2), the converse implication is not valid in general, though it is indeed the case if $\mathcal{M}$ is a finite von Neumann algebra and if, in addition, the norm and measure topologies coincide on $\mathcal{A}$ (see Proposition 6.6). This leads naturally to a study of spaces with the property that norm convergence of sequences is equivalent to weak convergence plus convergence for the measure topology. The study of such spaces seems to have been initiated in [29]. We show (Proposition 6.10) that, in such spaces, each relatively weakly compact subset is of uniformly absolutely continuous norm, provided the trace is finite and that this is no longer valid if the trace is not finite.

The final three sections give further applications to the study of various domination properties of completely positive operators between strongly symmetric spaces which complement results given in [18] and which depend crucially on the characterisation of sets of uniformly absolutely continuous norm given in Theorem 3.12. These theorems find their source in the study of positive operators on Banach lattices. A special case of Theorem 8.3 is that any completely positive operator on a strongly symmetric space with order continuous norm, which is dominated, in the sense of complete positivity, by a completely positive compact operator, has compact square. This is a non-commutative counterpart to a well-known theorem of Aliprantis and Burkinshaw [1] in the setting of Banach lattices. A particular consequence of Theorem 8.1 is that any completely positive operator on the predual of a finite von Neumann algebra, which is dominated by a completely positive Dunford-Pettis operator, is itself a Dunford-Pettis operator, and this continues to hold for all non-commutative Lorentz spaces and some Orlicz spaces. Results of this type go back to [17. The final section gives several "band-like" decomposition theorems for completely positive compact and Dunford-Pettis operators which again find their origins in the study of positive operators in Banach lattices [1, [17, [31, 45]. 


\section{Preliminaries And NOtation}

Throughout this paper $\mathcal{M} \subseteq \mathcal{B}(\mathcal{H})$ will denote a von Neumann algebra on some Hilbert space $\mathcal{H}$ (here, $\mathcal{B}(\mathcal{H})$ is the algebra of all bounded linear operators on $\mathcal{H}$ equipped with the operator norm denoted by $\|\cdot\|_{\infty}$ or $\left.\|\cdot\|_{\mathcal{B}(\mathcal{H})}\right)$. Unless otherwise stated, it will be assumed throughout that $\mathcal{M}$ is equipped with a fixed semifinite faithful normal trace $\tau$. For standard facts concerning von Neumann algebras, we refer to [11, [42]. The identity in $\mathcal{M}$ is denoted by $\mathbf{1}$, and we denote by $\mathcal{P}(\mathcal{M})$ the complete lattice of all (self-adjoint) projections in $\mathcal{M}$. A linear operator $x: \mathcal{D}(x) \rightarrow \mathcal{H}$, with domain $\mathcal{D}(x) \subseteq \mathcal{H}$, is said to be affiliated with $\mathcal{M}$ if $y x \subseteq x y$ for all $y$ in the commutant $\mathcal{M}^{\prime}$ of $\mathcal{M}$ (equivalently, $u x=x u$ for all unitary $u$ in $\left.\mathcal{M}^{\prime}\right)$. For any self-adjoint operator $x$ on $\mathcal{H}$, its spectral measure is denoted by $e^{x}$. A self-adjoint operator $x$ is affiliated with $\mathcal{M}$ if and only if $e^{x}(B) \in \mathcal{P}(\mathcal{M})$ for any Borel set $B \subseteq \mathbb{R}$.

The closed and densely defined operator $x$, affiliated with $\mathcal{M}$, is called $\tau$ measurable if and only if there exists a number $s \geq 0$ such that

$$
\tau\left(e^{|x|}(s, \infty)\right)<\infty
$$

The collection of all $\tau$-measurable operators is denoted by $S(\tau)$. With the sum and product defined as the respective closures of the algebraic sum and product, it is well known that $S(\tau)$ is a *algebra. For $\epsilon, \delta>0$, we denote by $V(\epsilon, \delta)$ the set of all $x \in S(\tau)$ for which there exists an orthogonal projection $p \in P(\mathcal{M})$ such that $p(\mathcal{H}) \subseteq \mathcal{D}(x),\|x p\|_{\mathcal{B}(\mathcal{H})} \leq \epsilon$ and $\tau(\mathbf{1}-p) \leq \delta$. The sets $\{V(\epsilon, \delta): \epsilon, \delta>0\}$ form a base at 0 for a metrizable Hausdorff topology on $S(\tau)$, which is called the measure topology. Equipped with this topology, $S(\tau)$ is a complete topological *-algebra. These facts and their proofs can be found in the papers [32, 43.

The collection of all closed, densely defined operators $x$ in $H$, affiliated with the von Neumann algebra $\mathcal{M}$ and satisfying $\tau\left(e^{|x|}(\lambda, \infty)\right)<\infty$ for all $\lambda>0$, will be denoted by $S_{0}(\tau)$. The elements of $S_{0}(\tau)$ are sometimes called $\tau$-compact operators. Evidently, each $x \in S_{0}(\tau)$ is $\tau$-measurable, that is, $S_{0}(\tau) \subseteq S(\tau)$.

For $x \in S(\tau)$, the singular value function $\mu(x)=\mu(\cdot ; x)=\mu(\cdot ;|x|)$ is defined by

$$
\mu(t ; x):=\inf \{s \geq 0: d(s ;|x|) \leq t\}, \quad t \geq 0,
$$

where

$$
d(s ;|x|):=\tau\left(e^{|x|}(s, \infty)\right), \quad s \geq 0 .
$$

It follows directly that the singular value function $\mu(x)$ is a decreasing, rightcontinuous function on the positive half-line $[0, \infty)$. Moreover, $\mu(u x v) \leq$ $\|u\|_{\infty}\|v\|_{\infty} \mu(x)$ for all $u, v \in \mathcal{M}$ and $x \in S(\tau)$ and $\mu(f(x))=f(\mu(x))$ whenever $0 \leq x \in S(\tau)$ and $f$ is an increasing continuous function on $[0, \infty)$ which satisfies $f(0)=0$.

It should be observed that a sequence $\left\{x_{n}\right\}_{n=1}^{\infty}$ in $S(\tau)$ converges to zero for the measure topology if and only if $\mu\left(t ; x_{n}\right) \rightarrow 0$ as $n \rightarrow \infty$ for all $t>0$.

If $m$ denotes Lebesgue measure on the semiaxis [0, $\infty)$ and if we consider $L^{\infty}(m)$ as an Abelian von Neumann algebra acting via multiplication on the Hilbert space $\mathcal{H}=L^{2}(m)$, with the trace given by integration with respect to $m$, then $S(m)$ consists of all measurable functions on $[0, \infty)$ which are bounded except on a set of finite measure. In this case for $f \in S(m)$, the generalized singular value function $\mu(f)$ is precisely the classical decreasing rearrangement of the function $|f|$, which 
is usually denoted by $f^{*}$. In this setting, convergence for the measure topology coincides with the usual notion of convergence in measure.

If $\mathcal{M}=\mathcal{B}(\mathcal{H})$ and $\tau$ is the standard trace, then $S(\tau)=\mathcal{M}$, the measure topology coincides with the operator norm topology.

If $x \in S(\tau)$, then $x$ is compact if and only if $\lim _{t \rightarrow \infty} \mu(t ; x)=0$; in this case,

$$
\mu_{n}(x)=\mu(t ; x), \quad t \in[n, n+1), \quad n=0,1,2, \ldots,
$$

and the sequence $\left\{\mu_{n}(x)\right\}_{n=0}^{\infty}$ is just the sequence of eigenvalues of $|x|$ in nonincreasing order and counted according to multiplicity.

The real vector space $S_{h}(\tau)=\left\{x \in S(\tau): x=x^{*}\right\}$ is a partially ordered vector space with the ordering defined by setting $x \geq 0$ if and only if $\langle x \xi, \xi\rangle \geq 0$ for all $\xi \in \mathcal{D}(x)$. The positive cone in $S_{h}(\tau)$ will be denoted by $S(\tau)^{+}$. If $0 \leq x_{\alpha} \uparrow_{\alpha} \leq x$ holds in $S(\tau)^{+}$, then $\sup _{\alpha} x_{\alpha}$ exists in $S(\tau)^{+}$. The trace $\tau$ extends to $S(\tau)^{+}$as a non-negative extended real-valued functional which is positively homogeneous, additive, unitarily invariant and normal. This extension is given by

$$
\tau(x)=\int_{0}^{\infty} \mu(t ; x) d t, \quad x \in S(\tau)^{+},
$$

and satisfies $\tau\left(x^{*} x\right)=\tau\left(x x^{*}\right)$ for all $x \in S(\tau)$. It should be observed that if $f$ is an increasing continuous function on $[0, \infty)$ satisfying $f(0)=0$, then

$$
\tau(f(|x|))=\int_{0}^{\infty} \mu(t ; f(|x|)) d t=\int_{0}^{\infty} f(\mu(t ; x)) d t
$$

for all $x \in S(\tau)$.

If $1 \leq p<\infty$, we set $L^{p}(\tau)=\left\{x \in S(\tau): \tau\left(|x|^{p}\right)<\infty\right\}$. Note that it follows from (2.1) that $L^{p}(\tau)$ is also given by

$$
L^{p}(\tau)=\left\{x \in S(\tau): \mu(x) \in L^{p}(m)\right\} .
$$

The space $L^{p}(\tau)$ is a linear subspace of $S(\tau)$ and the functional $x \longmapsto\|x\|_{L^{p}(\tau)}=$ $\tau\left(|x|^{p}\right)^{1 / p}, x \in L^{p}(\tau)$, defines a norm on $L^{p}(\tau)$. It should be observed that $\|x\|_{L^{p}(\tau)}=\|\mu(x)\|_{L^{p}(m)}$ for all $x \in L^{p}(\tau)$. Equipped with this norm, $L^{p}(\tau)$ is a Banach space. In this setting, we also have that $L^{\infty}(\tau)=\mathcal{M}$.

In the commutative setting, the spaces $L^{p}(\tau)$ are the familiar Lebesgue spaces. In the special case that $\mathcal{M}$ is $\mathcal{B}(\mathcal{H})$ equipped with standard trace, the corresponding $L^{p}$-spaces are the Schatten classes $\mathfrak{S}_{p}$. As is well known, the space $L^{1}(\tau)$ may be identified with the von Neumann algebra predual of $\mathcal{M}$ with respect to trace duality.

If $(\mathcal{N}, \sigma)$ is a semifinite von Neumann algebra, if $x \in S(\tau)$ and $y \in S(\sigma)$, then $x$ is said to be submajorised by $y$ (in the sense of Hardy, Littlewood and Polya) if and only if

$$
\int_{0}^{t} \mu(s ; x) d s \leq \int_{0}^{t} \mu(s ; y) d s
$$

for all $t \geq 0$. We write $x \prec \prec y$ or, equivalently, $\mu(x) \prec \prec \mu(y)$.

It will be convenient to adopt the following terminology. A linear subspace $E \subseteq S(\tau)$, equipped with a norm $\|\cdot\|_{E}$, will be called

(i) symmetrically normed if $x \in E, y \in S(\tau)$ and $\mu(y) \leq \mu(x)$ imply that $y \in E$ and $\|y\|_{E} \leq\|x\|_{E}$

(ii) strongly symmetrically normed if $E$ is symmetrically normed and its norm has the additional property that $\|y\|_{E} \leq\|x\|_{E}$ whenever $x, y \in E$ satisfy $y \prec \prec x$. 
In the present paper we shall only consider strongly symmetrically normed spaces. It should be pointed out that all results are also valid for symmetrically normed spaces if one assumes in addition that the von Neumann algebra $\mathcal{M}$ is either non-atomic (that is, does not contain any minimal projections) or atomic and all minimal projections have equal trace (see [21, 20]).

If a (strongly) symmetrically normed space is Banach, then it will be simply called a (strongly) symmetric space.

If $x \in S(\tau)$, then the projection onto the closure of the range of $|x|$ is called the support of $x$ and is denoted by $s(x)$. We set $\mathcal{F}(\tau):=\{x \in \mathcal{M}: \tau(s(x))<\infty\}$.

It may be shown that any strongly symmetrically normed space $E$ for which $\bigvee_{x \in E} s(x)=\mathbf{1}$ (which will always be assumed) satisfies

$$
\mathcal{F}(\tau) \subseteq E \subseteq L^{1}(\tau)+\mathcal{M}
$$

with continuous inclusions (where $\mathcal{F}(\tau)$ is equipped with the $L^{1} \cap L^{\infty}$-norm). If, in addition, $E$ is a Banach space, then $L^{1}(\tau) \cap \mathcal{M} \subseteq E$, with continuous embedding.

Remark 2.1. We point out that the terminology introduced above differs from that which has been used elsewhere in the literature. We point out explicitly that the terms "symmetrically normed" and "strongly symmetrically normed" as defined above are used in the present paper instead of the earlier terminology of "rearrangement-invariant" and "symmetrically normed", respectively, used in the papers [14], 15].

If $\mathcal{M}$ is $L^{\infty}(m)$, then a symmetrically normed space $E \subseteq S(m)$ will be called, for simplicity, a symmetrically normed space on $[0, \infty)$.

If $E \subseteq S(\tau)$ is a strongly symmetrically normed space, then the embedding of $E$ into $S(\tau)$ is continuous from the norm topology of $E$ to the measure topology on $S(\tau)$.

A wide class of non-commutative strongly symmetrically normed spaces may be constructed as follows. If $E \subseteq S(m)$ is a strongly symmetrically normed space on $[0, \infty)$, set

$$
E(\tau)=\{x \in S(\tau): \mu(x) \in E\}, \quad\|x\|_{E(\tau)}:=\|\mu(x)\|_{E} .
$$

It may be shown as in [12], 13], 41] that $\left(E(\tau),\|\cdot\|_{E(\tau)}\right)$ is strongly symmetrically normed and is a Banach space if $E$ is a Banach space. See also [27.

Now suppose that $E \subseteq S(\tau)$ is a strongly symmetrically normed space and let

$$
E^{\times}=\left\{y \in S(\tau): \sup \left\{\tau(|x y|): x \in E,\|x\|_{E} \leq 1\right\}<\infty\right\}
$$

and

$$
\|y\|_{E^{\times}}=\sup \left\{\tau(|x y|): x \in E,\|x\|_{E} \leq 1\right\} .
$$

If $y \in S(\tau)$, then $y \in E^{\times}$if and only if

$$
\sup \left\{\int_{[0, \infty)} \mu(x) \mu(y) d m: x \in E,\|x\|_{E} \leq 1\right\}<\infty,
$$

in which case, the latter quantity is equal to $\|y\|_{E^{\times}}$. The space $\left(E^{\times},\|\cdot\|_{E^{\times}}\right)$is a strongly symmetrically normed space and is called the Köthe dual of $E$. The symmetrically normed space $E$ is said to have the Fatou property if, whenever $0 \leq x_{\alpha} \uparrow_{\alpha} \subseteq E$ is an upwards directed system with $\sup \left\|x_{\alpha}\right\|_{E}<\infty$, it follows that 
$x=\sup _{\alpha} x_{\alpha}$ exists in $E$ and $\|x\|_{E}=\sup _{\alpha}\left\|x_{\alpha}\right\|_{E}$. The Köthe dual $E^{\times}$of any strongly symmetrically normed space $E \subseteq S(\tau)$ always has the Fatou property. If $y \in E^{\times}$, set $\phi_{y}: E \rightarrow \mathbb{C}, \phi_{y}(x)=\tau(x y), x \in E$. The mapping $y \rightarrow \phi_{y}, y \in E^{\times}$, is an isometry of $E^{\times}$into the Banach dual $E^{*}$. The linear functional $\phi$ on the symmetrically normed space $E \subseteq S(\tau)$ is said to be normal if whenever $x_{\alpha} \downarrow_{\alpha}$ $0 \subseteq E$, it follows that $\phi\left(x_{\alpha}\right) \rightarrow_{\alpha} 0$ and singular if $\phi$ vanishes on some order dense ideal in $E$ (see e.g [16, 19]). The space of all normal (respectively, singular) linear functionals on $E$ is denoted by $E_{n}^{*}$ (respectively, $E_{s}^{*}$ ). It is shown in [19] that if $E \subseteq S(\tau)$ is strongly symmetric, then the Banach dual $E^{*}$ admits the unique decomposition $E^{*}=E_{n}^{*} \oplus E_{s}^{*}$, called the Yosida-Hewitt decomposition, and that $E_{n}^{*}$ may be identified with the Köthe dual $E^{\times}$by trace duality (see also [15]). It is shown in [19] that the natural projections $P_{n}: E^{*} \rightarrow E_{n}^{*}, P_{s}: E^{*} \rightarrow E_{s}^{*}$ are positive, bounded projections which are sequentially continuous for the weak *topology $\sigma\left(E^{*}, E\right)$. The norm on $E$ is said to be order continuous if $\left\|x_{\alpha}\right\|_{E} \downarrow_{\alpha} 0$ whenever $x_{\alpha} \downarrow_{\alpha} 0 \subseteq E$. The norm on $E$ is order continuous if and only if every continuous linear functional on $E$ is normal, and in this case, the Banach dual $E^{*}$ may be identified with the Köthe dual $E^{\times}[15$, [21. See also 20].

\section{SEtS OF UNIFORMLY ABSOLUtely CONTINUOUS NORM}

Let $E \subseteq S(\tau)$ be a symmetrically normed space. If $x \in E$, then $x$ is said to have absolutely continuous norm if and only if for all decreasing sequences $\left\{e_{n}\right\}_{n=1}^{\infty}$ in $P(\mathcal{M})$

$$
e_{n} \downarrow_{n} 0 \Longrightarrow\left\|e_{n} x e_{n}\right\|_{E} \rightarrow_{n} 0 .
$$

We shall need the following theorem, which collects together a number of useful characterisations of elements of absolutely continuous norm (see e.g. 19], Theorem 6.13, and also [7, [15]).

Theorem 3.1. Suppose that $E \subseteq S(\tau)$ is a strongly symmetrically normed space. If $x \in E$, then the following statements are equivalent:

(i) $x$ has absolutely continuous norm;

(ii) for all decreasing systems $\left\{e_{\alpha}\right\}$ in $P(\mathcal{M})$,

$$
e_{\alpha} \downarrow_{\alpha} 0 \Longrightarrow\left\|e_{\alpha} x e_{\alpha}\right\|_{E} \rightarrow_{\alpha} 0
$$

(iii) for all decreasing sequences $\left\{e_{n}\right\}_{n=1}^{\infty}$ in $P(\mathcal{M})$,

$$
e_{n} \downarrow_{n} 0 \Longrightarrow\left\|x e_{n}\right\|_{E} \rightarrow_{n} 0 ;
$$

(iv) for all decreasing systems $\left\{e_{\alpha}\right\}$ in $P(\mathcal{M})$,

$$
e_{\alpha} \downarrow_{\alpha} 0 \Longrightarrow\left\|x e_{\alpha}\right\|_{E} \rightarrow_{\alpha} 0
$$

(v) $x$ has order continuous norm, that is, for all decreasing systems $\left\{x_{\alpha}\right\}$ in $E$,

$$
|x| \geq x_{\alpha} \downarrow_{\alpha} 0 \Longrightarrow\left\|x_{\alpha}\right\|_{E} \rightarrow_{\alpha} 0 ;
$$

(vi) for all decreasing sequences $\left\{x_{n}\right\}_{n=1}^{\infty}$ in $E$,

$$
|x| \geq x_{n} \downarrow_{n} 0 \Longrightarrow\left\|x_{n}\right\|_{E} \rightarrow_{n} 0 .
$$


Moreover, if $E$ is a strongly symmetric space, then the above conditions are also equivalent with each of the following two statements:

(vii) for all mutually orthogonal sequences $\left\{e_{n}\right\}_{n=1}^{\infty}$ in $P(\mathcal{M})$ it follows that

$$
\left\|x e_{n}\right\|_{E} \rightarrow_{n} 0
$$

(viii) for all mutually orthogonal sequences $\left\{e_{n}\right\}_{n=1}^{\infty}$ in $P(\mathcal{M})$ it follows that

$$
\left\|e_{n} x e_{n}\right\|_{E} \rightarrow_{n} 0 .
$$

Analogously to the Banach lattice setting, the set of all elements of $E$ with absolutely continuous norm will be denoted by $E^{a n}$.

Proposition 3.2. If $E \subseteq S(\tau)$ is strongly symmetric and $\mathcal{A} \subseteq E$, then the following statements are equivalent.

(i)

$$
\sup _{x \in \mathcal{A}}\left\|e_{n} x e_{n}\right\|_{E} \rightarrow_{n} 0, \quad \forall e_{n} \downarrow_{n} 0 \subseteq P(\mathcal{M})
$$

(ii)

$$
\sup _{x \in \mathcal{A}}\left\|e_{n} x e_{n}\right\|_{E} \rightarrow_{n} 0
$$

for all mutually disjoint sequences $\left\{e_{n}\right\}_{n \geq 1} \subseteq P(\mathcal{M})$;

$$
\sup _{x \in \mathcal{A}}\left\|e_{\alpha} x e_{\alpha}\right\|_{E} \rightarrow_{\alpha} 0
$$

for every downwards directed system $e_{\alpha} \downarrow_{\alpha} 0 \subseteq P(\mathcal{M})$.

Proof. Note that it follows from Theorem 3.1 that each of the above conditions implies $\mathcal{A} \subseteq E^{a n}$. The implication (iii) $\Longrightarrow$ (i) is clear. To see that (i) $\Longrightarrow$ (ii), suppose that $\left\{e_{n}\right\}_{n \geq 1}$ is a mutually disjoint sequence of projections but that there exist $\epsilon>0$ and a sequence $\left\{x_{n}\right\}_{n \geq 1} \subseteq \mathcal{A}$ such that

$$
\left\|e_{n} x_{n} e_{n}\right\|_{E} \geq \epsilon, \quad \forall n \geq 1 \text {. }
$$

Set $f_{n}:=\sup _{m>n} e_{m}$. Observe that $f_{n} \downarrow_{n} 0 \subseteq P(\mathcal{M})$, and so it follows from the assumption (i) that $\sup _{x \in \mathcal{A}}\left\|f_{n} x f_{n}\right\|_{E} \rightarrow_{n} 0$. On the other hand,

$$
\epsilon \leq\left\|e_{n} x_{n} e_{n}\right\|_{E} \leq\left\|e_{n} f_{n} x_{n} f_{n} e_{n}\right\|_{E} \leq\left\|f_{n} x_{n} f_{n}\right\|_{E}
$$

for all $n \geq 1$ and this is a contradiction.

To show that (ii) $\Longrightarrow($ iii), suppose that (iii) fails for some downwards directed system $e_{\alpha} \downarrow_{\alpha} 0 \subseteq P(\mathcal{M})$. In particular, there exists $\epsilon>0$ such that, for every index $\alpha$, there exists an index $\beta$ and an element $x_{\beta} \in \mathcal{A}$ such that $e_{\beta} \leq e_{\alpha}$ and $\left\|e_{\beta} x_{\beta} e_{\beta}\right\| \geq \epsilon$. Using this and the characterisation given by Theorem [3.1(iv) and (viii) (applied to $x$ and $x^{*}$ ) of absolutely continuous elements in $E$, it follows by a simple inductive argument that there exist sequences $\left\{e_{\alpha(i)}\right\}_{i \geq 1} \subseteq\left\{e_{\alpha}\right\},\left\{x_{i}\right\}_{i \geq 1} \subseteq$ $\mathcal{A}$ such that $e_{\alpha(i)} \downarrow_{i}$,

$$
\left\|e_{\alpha(i)} x_{i} e_{\alpha(i)}\right\|_{E} \geq \epsilon, \quad i \geq 1
$$

and

$$
\left\|x_{i} e_{\alpha(j)}\right\|_{E} \leq \epsilon / 4, \quad\left\|e_{\alpha(j)} x_{i}\right\|_{E} \leq \epsilon / 4, \quad \forall j>i .
$$


Now observe that, for all $i \geq 1$,

$$
\begin{aligned}
\left\|\left(e_{\alpha(i)}-e_{\alpha(i+1)}\right) x_{i}\left(e_{\alpha(i)}-e_{\alpha(i+1)}\right)\right\|_{E} & \geq\left\|e_{\alpha(i)} x_{i} e_{\alpha(i)}\right\|_{E}-\left\|e_{\alpha(i)} x_{i} e_{\alpha(i+1)}\right\|_{E} \\
& -\left\|e_{\alpha(i+1)} x_{i} e_{\alpha(i)}\right\|_{E} \\
& -\left\|e_{\alpha(i+1)} x_{i} e_{\alpha(i+1)}\right\|_{E} \\
& \geq\left\|e_{\alpha(i)} x_{i} e_{\alpha(i)}\right\|_{E}-\left\|x_{i} e_{\alpha(i+1)}\right\|_{E} \\
& -2\left\|e_{\alpha(i+1)} x_{i}\right\|_{E} \\
& \geq \epsilon / 4 .
\end{aligned}
$$

Since the sequence $\left\{e_{\alpha(i)}-e_{\alpha(i+1)}\right\}_{i \geq 1} \subseteq P(\mathcal{M})$ is pairwise disjoint, this contradicts (ii) and so the implication (ii) $\Longrightarrow$ (iii) is established.

Definition 3.3. If $\mathcal{A} \subseteq E$ is a bounded set, then $\mathcal{A}$ will be said to be of uniformly absolutely continuous norm if $\mathcal{A}$ satisfies one (and hence all) of the equivalent statements of the preceding Proposition 3.2 .

Note that in the Banach lattice setting, sets with property (ii) of the preceding proposition coincide with the class of $L$-weakly compact sets (see e.g. 31, Definition 3.6.1).

The following result is proved in [19, Theorem 6.11 (see also [40]).

Theorem 3.4. Suppose that $E \subseteq S(\tau)$ is strongly symmetrically normed. If $\left\{x_{n}\right\}_{n=1}^{\infty} \subseteq E^{a n}$, then the following statements are equivalent.

(i) $\left\|x_{n}\right\|_{E} \rightarrow 0$ as $n \rightarrow \infty$.

(ii) $x_{n} \rightarrow_{n} 0$ for the measure topology, and the set $\left\{x_{n}: n \in \mathbb{N}\right\}$ is of uniformly absolutely continuous norm.

It is worth noting that the implication (ii) $\Longrightarrow$ (i) remains valid in the case of nets.

Corollary 3.5. Suppose that $E \subseteq S(\tau)$ is strongly symmetrically normed and that $\left\{x_{\alpha}\right\}_{\alpha \in \mathbb{A}} \subseteq E^{a n}$ is a net. If $x_{\alpha} \rightarrow_{\alpha} 0$ for the measure topology and if the set $\left\{x_{\alpha}: \alpha \in \mathbb{A}\right\}$ is of uniformly absolutely continuous norm, then $\left\|x_{\alpha}\right\|_{E} \rightarrow_{\alpha} 0$.

Proof. If it is not the case that $\left\|x_{\alpha}\right\|_{E} \rightarrow_{\alpha} 0$, then passing to a subnet and relabelling if necessary, it may be assumed that there exists $0<c \in(0, \infty)$ such that $\lim \sup _{\alpha}\left\|x_{\alpha}\right\|_{E} \geq c$. From the definition of convergence in measure and using the fact that the index set $\mathbb{A}$ is a directed set, it follows that there exists an increasing sequence of indices $\{\alpha(n)\}_{n=1}^{\infty}$ such that $x_{\alpha(n)} \in V(1 / n, 1 / n)$ and $\left\|x_{\alpha(n)}\right\|_{E} \geq c-$ $1 / n$. By Theorem 3.4 it follows that $\left\|x_{\alpha(n)}\right\|_{E} \rightarrow_{n} 0$, and this yields a contradiction.

Recall that $\mathcal{F}(\tau):=\{x \in \mathcal{M}: \tau(s(x))<\infty\}$. If $\mathcal{M}=\mathcal{B}(\mathcal{H})$, then $\mathcal{F}(\tau)$ is simply the ideal of finite rank operators.

Proposition 3.6. If $E \subseteq S(\tau)$ is strongly symmetrically normed and if $x \in E$, then the following statements are equivalent.

(i) $x \in E^{a n}$.

(ii) Whenever $\left\{x_{\alpha}\right\} \subset \mathcal{M}_{+}$is a bounded net which converges to 0 for the weak* topology $\sigma\left(\mathcal{M}, L^{1}(\tau)\right)$, it follows that $\left\|x_{\alpha} x\right\|_{E} \rightarrow_{\alpha} 0$. 
Proof. (i) $\Longrightarrow$ (ii) It clearly may be assumed that $x \geq 0$. It is well known (see e.g. [15, 21]) that every $x \in E^{a n}$ belongs to the norm closure of the set $\mathcal{F}(\tau)$, and therefore, via a standard argument, it may be assumed that $0 \leq x \in \mathcal{F}(\tau)$. From the assumption that $0 \leq x_{\alpha} \rightarrow_{\alpha} 0$ for the weak*-topology $\sigma\left(\mathcal{M}, L^{1}(\tau)\right)$, it follows that

$$
\left\|x^{1 / 2} x_{\alpha} x^{1 / 2}\right\|_{L^{1}(\tau)}=\tau\left(x^{1 / 2} x_{\alpha} x^{1 / 2}\right)=\tau\left(x_{\alpha} x\right) \rightarrow 0
$$

as $n \rightarrow \infty$. Consequently, $x^{1 / 2} x_{\alpha} x^{1 / 2} \rightarrow_{\alpha} 0$ for the measure topology. It may clearly be assumed further that $0 \leq x_{\alpha} \leq \mathbf{1}$ for all $\alpha$ so that $0 \leq x^{1 / 2} x_{\alpha} x^{1 / 2} \leq x$ for all $\alpha$. This implies that $\left\{x^{1 / 2} x_{\alpha} x^{1 / 2}\right\} \subseteq E$ is of uniformly absolutely continuous norm. By Corollary [3.5, it follows that $\left\|x^{1 / 2} x_{\alpha} x^{1 / 2}\right\|_{E} \rightarrow_{\alpha} 0$. Using the estimate given in [19], Lemma 4.4 (or in [7], Lemma 2.3), and the fact that $0 \leq x_{\alpha}^{1 / 2} \leq \mathbf{1}$ for all $\alpha$, it follows that

$$
\left\|x_{\alpha} x\right\|_{E} \leq\left\|x x_{\alpha}^{1 / 2}\right\|_{E} \leq K\|x\|_{E}^{1 / 2}\left\|x^{1 / 2} x_{\alpha} x^{1 / 2}\right\|_{E}^{1 / 2} \rightarrow_{\alpha} 0 .
$$

(ii) $\Longrightarrow$ (i). This implication follows immediately from the fact that if $\left\{e_{n}\right\}_{n=1}^{\infty} \subseteq$ $P(\mathcal{M})$ satisfies $e_{n} \downarrow_{n} 0$, then $e_{n} \rightarrow_{n} 0$ for the weak* topology $\sigma\left(\mathcal{M}, L_{1}(\tau)\right)$.

Lemma 3.7. If $x=x^{*} \in S(\tau)$ and if $\delta>0$, then there exists a projection $e \in$ $P(\mathcal{M})$ such that $e x=x e, \tau(e) \leq \delta$ and

$$
\|x(\mathbf{1}-e)\|_{\infty} \leq \mu(\delta ; x) .
$$

Proof. Given $\delta>0$, set $\alpha=\mu(\delta ; x)$ and note that $d(\alpha ;|x|) \leq \delta$. Defining $e:=$ $e^{|x|}(\alpha, \infty)$, it follows that

$$
\|x(\mathbf{1}-e)\|_{\infty}=\||x|(\mathbf{1}-e)\|_{\infty}=\left\||x| e^{|x|}[0, \alpha]\right\|_{\infty} \leq \alpha=\mu(\delta ; x)
$$

and $\tau(e)=d(\alpha ;|x|) \leq \delta$. Finally, since

$$
e=e^{|x|}(\alpha, \infty)=e^{x}(-\infty,-\alpha)+e^{x}(\alpha, \infty),
$$

it follows that $x e=e x$.

Lemma 3.8. Let $E \subseteq S(\tau)$ be a strongly symmetrically normed space and let $K$ be the embedding constant of $E$ into $L^{1}(\tau)+\mathcal{M}$. If $\mathcal{A} \subseteq E$ is bounded and if $\delta>0$, then

$$
\sup _{x \in \mathcal{A}} \mu(\delta ; x) \leq K \min \{\delta, 1\}^{-1} \sup _{x \in \mathcal{A}}\|x\|_{E} .
$$

Proof. Observe that

$$
\chi_{[0, \delta)} \mu(\delta ; x) \leq \mu(x)
$$

so that

$$
\mu(\delta ; x)\left\|\chi_{[0, \delta)}\right\|_{L^{1}+L^{\infty}} \leq\|\mu(x)\|_{L^{1}+L^{\infty}}=\|x\|_{L^{1}(\tau)+\mathcal{M}} \leq K\|x\|_{E},
$$

and the assertion follows directly since

$$
\left\|\chi_{[0, \delta)}\right\|_{L^{1}+L^{\infty}}=\int_{0}^{1} \chi_{[0, \delta)} d m=\min \{\delta, 1\} .
$$

Definition 3.9. For all $x \in E$ and $\delta>0$, set

$$
\omega(x ; \delta)=\sup \left\{\|e x e\|_{E}: e \in P(\mathcal{M}), \tau(e) \leq \delta\right\} .
$$


Observe that $\omega(x ; \delta)<\infty$ for every $x \in E$ and $\delta>0$. Furthermore, when the $\left(E(\tau),\|\cdot\|_{E(\tau)}\right)$ is a strongly symmetrically Banach space generated by a strongly symmetrical space $E \subseteq S(m)$ (see the Introduction), then $\omega(x ; \delta) \leq\left\|\mu(x) \chi_{[0, \delta]}\right\|_{E}$.

Lemma 3.10. If $E \subseteq S(\tau)$ is strongly symmetric and if $\mathcal{A} \subseteq E$ is of uniformly absolutely continuous norm, then

$$
\lim _{\delta \rightarrow 0} \sup _{x \in \mathcal{A}} \omega(x ; \delta)=0 .
$$

Proof. It clearly suffices to show that

$$
\lim _{n \rightarrow \infty} \sup _{x \in \mathcal{A}} \omega\left(x ; 2^{-n}\right)=0 .
$$

For each $n \in \mathbb{N}$, there exists a projection $e_{n}$ with $\tau\left(e_{n}\right) \leq 2^{-n}$, and there exists $x_{n} \in \mathcal{A}$ such that

$$
\left\|e_{n} x_{n} e_{n}\right\|_{E} \geq \sup _{x \in \mathcal{A}} \omega\left(x ; 2^{-n}\right)-1 / n \text {. }
$$

Set $f_{n}=\sup _{m \geq n} e_{m}$ and observe that $\tau\left(f_{n}\right) \leq 2^{-n+1}$. This implies that $f_{n} \downarrow_{n} 0$. It follows that

$$
\left\|e_{n} x_{n} e_{n}\right\|_{E} \leq\left\|f_{n} x_{n} f_{n}\right\|_{E} \leq \sup _{x \in \mathcal{A}}\left\|f_{n} x f_{n}\right\|_{E} .
$$

Since $\mathcal{A}$ is of uniformly absolutely continuous norm, it follows that

$$
\sup _{x \in \mathcal{A}}\left\|f_{n} x f_{n}\right\|_{E} \rightarrow_{n} 0
$$

and so $\left\|e_{n} x_{n} e_{n}\right\|_{E} \rightarrow_{n} 0$. It now follows also that $\sup _{x \in \mathcal{A}} \omega\left(x ; 2^{-n}\right) \rightarrow_{n} 0$, and this establishes the lemma.

Corollary 3.11. Suppose that $E \subseteq S(\tau)$ is strongly symmetric. If $\tau(\mathbf{1})<\infty$, then the following statements are equivalent for a subset $\mathcal{A} \subseteq E$.

(i) $\mathcal{A}$ is of uniformly absolutely continuous norm.

(ii)

$$
\lim _{\delta \rightarrow 0} \sup _{x \in \mathcal{A}} \omega(x ; \delta)=0 .
$$

Proof. The implication (i) $\Longrightarrow$ (ii) follows from Lemma 3.10 To show the implication (ii) $\Longrightarrow$ (i), suppose that $e_{n} \downarrow_{n} 0 \subseteq P(\mathcal{M})$. Since $\tau(\mathbf{1})<\infty$, it follows that $\tau\left(e_{n}\right) \rightarrow$ 0 . Consequently, if $\delta>0$, there exists $N \in \mathbb{N}$ such that $\tau\left(e_{n}\right)<\delta$ for all $n \geq N$. It follows that

$$
\sup _{x \in \mathcal{A}}\left\|e_{n} x e_{n}\right\|_{E} \leq \sup _{x \in \mathcal{A}} \omega(x ; \delta), \quad n \geq \mathbb{N}
$$

and this suffices to complete the proof.

If $X$ is a Banach space, the unit ball of $X$ will be denoted by $B(X)$. The following result yields an important characterisation of the sets with uniformly absolutely continuous norm.

Theorem 3.12. Let $E \subseteq S(\tau)$ be a symmetric space with order continuous norm. If $\mathcal{A} \subseteq E$ is bounded, then the following statements are equivalent.

(i) $\mathcal{A}$ is of uniformly absolutely continuous norm.

(ii) For every upwards directed net of $\tau$-finite trace projections $\left\{e_{\alpha}\right\} \uparrow \mathbf{1} \subseteq$ $P(\mathcal{M})$ and every $\epsilon>0$, there exists such $\alpha$ and a constant $C_{\epsilon}>0$ that

$$
\mathcal{A} \subseteq C_{\epsilon}\left(e_{\alpha} B(\mathcal{M})+B(\mathcal{M}) e_{\alpha}\right)+\epsilon B(E) .
$$


(iii) For every $\epsilon>0$, there exists $0 \leq y_{\epsilon} \in \mathcal{F}(\tau)$ such that

$$
\mathcal{A} \subseteq y_{\epsilon} B(\mathcal{M})+B(\mathcal{M}) y_{\epsilon}+\epsilon B(E) .
$$

(iv)

$$
\sup _{x \in \mathcal{A}}\left\|x_{\alpha} x y_{\alpha}\right\|_{E} \rightarrow_{\alpha} 0
$$

for all bounded nets $\left\{x_{\alpha}\right\},\left\{y_{\alpha}\right\} \subseteq \mathcal{M}_{+}$such that $x_{\alpha}, y_{\alpha} \rightarrow_{\alpha} 0$ for the strong operator topology.

Proof. (i) $\Longrightarrow$ (ii) Let $\epsilon>0$ be given and let $\mathcal{A}$ be of uniformly absolutely continuous norm. Fix the upwards directed net of $\tau$-finite trace projections $\left\{e_{\alpha}\right\} \uparrow$ $\mathbf{1} \subseteq P(\mathcal{M})$ and consider the downwards directed net $\left\{f_{\alpha}:=\mathbf{1}-e_{\alpha}\right\} \downarrow 0$. By Proposition 3.2 (iii), there exists $\alpha$ such that the projection $f:=f_{\alpha} \in P(\mathcal{M})$ with $\tau(\mathbf{1}-f)<\infty$ satisfies

$$
\sup _{x \in \mathcal{A}}\|f x f\|_{E}<\epsilon / 4 \text {. }
$$

Since $\mathcal{A}^{*}=\left\{x^{*}: x \in \mathcal{A}\right\}$ is also of uniformly absolutely continuous norm, it follows that the sets of real and imaginary parts of elements of $\mathcal{A}$ are of uniformly absolutely continuous norm. It follows that it suffices to prove the implication for the case when $\mathcal{A}$ consists of self-adjoint elements. Suppose $x=x^{*} \in \mathcal{A}$ and let $\delta>0$ be given. By Lemma 3.7, there exists $e \in P(\mathcal{M})$ such that

$$
x e=e x, \quad \tau(e) \leq \delta, \quad\|x(\mathbf{1}-e)\|_{\infty} \leq \mu(\delta ; x) .
$$

It is clear that

$$
x=(\mathbf{1}-f) x f+(\mathbf{1}-f) x(\mathbf{1}-f)+f x(\mathbf{1}-f)+f x f .
$$

Now,

$$
\begin{aligned}
(\mathbf{1}-f) x f & =(\mathbf{1}-f) x e f+(\mathbf{1}-f) x(\mathbf{1}-e) f, \\
(\mathbf{1}-f) x(\mathbf{1}-f) & =(\mathbf{1}-f) x e(\mathbf{1}-f)+(\mathbf{1}-f) x(\mathbf{1}-e)(\mathbf{1}-f), \\
f x(\mathbf{1}-f) & =f x e(\mathbf{1}-f)+f x(\mathbf{1}-e)(\mathbf{1}-f) .
\end{aligned}
$$

Using the fact that $x e=e x=e x e$, observe that

$$
\|(\mathbf{1}-f) x e f\|_{E} \leq\|x e\|_{E}=\| \text { exe } \|_{E} \leq \omega(\delta ; x)
$$

and similarly

$$
\|(\mathbf{1}-f) x e(\mathbf{1}-f)\|_{E} \leq \omega(\delta ; x), \quad\|f x e(\mathbf{1}-f)\|_{E} \leq \omega(\delta ; x) .
$$

Now observe further that $x(\mathbf{1}-e) f \in \mu(\delta ; x) B(\mathcal{M})$ and so

$$
\begin{gathered}
(\mathbf{1}-f) x(\mathbf{1}-e) f \in \mu(\delta ; x)(\mathbf{1}-f) B(\mathcal{M}) \subseteq(1 / 2) C_{\epsilon}(\mathbf{1}-f) B(\mathcal{M}), \\
(\mathbf{1}-f) x(\mathbf{1}-e)(\mathbf{1}-f) \in \mu(\delta ; x)(\mathbf{1}-f) B(\mathcal{M}) \subseteq(1 / 2) C_{\epsilon}(\mathbf{1}-f) B(\mathcal{M}), \\
f x(\mathbf{1}-e)(\mathbf{1}-f) \in \mu(\delta ; x) B(\mathcal{M})(\mathbf{1}-f) \subseteq(1 / 2) C_{\epsilon} B(\mathcal{M})(\mathbf{1}-f),
\end{gathered}
$$

where

$$
C_{\epsilon}:=2 \sup _{x \in \mathcal{A}} \mu(\delta ; x)<\infty
$$

by Lemma 3.8. By Lemma 3.10, it may be assumed that $\delta>0$ satisfies

$$
\sup _{x \in \mathcal{A}} \omega(\delta ; x)<\epsilon / 4 .
$$


If we set

$$
x_{\epsilon}=(\mathbf{1}-f) x(\mathbf{1}-e) f+(\mathbf{1}-f) x(\mathbf{1}-e)(\mathbf{1}-f)+f x(\mathbf{1}-e)(\mathbf{1}-f),
$$

then

$$
x_{\epsilon} \in C_{\epsilon}((\mathbf{1}-f) B(\mathcal{M})+B(\mathcal{M})(\mathbf{1}-f))
$$

and

$$
\left\|x-x_{\epsilon}\right\|_{E} \leq 3 \omega(\delta ; x)+\epsilon / 4 \leq \epsilon .
$$

The assertion of (ii) now follows.

The assertion (ii) $\Longrightarrow$ (iii) is trivial.

(iii) $\Longrightarrow$ (iv) Let $\epsilon>0$ be given. From (iii), there exists $y_{\epsilon} \in \mathcal{F}(\tau)$ such that

$$
\mathcal{A} \subseteq y_{\epsilon} B(\mathcal{M})+B(\mathcal{M}) y_{\epsilon}+\epsilon B(E) .
$$

Suppose that $0 \leq x_{\alpha}, y_{\alpha} \in B(\mathcal{M})$ are bounded nets such that $x_{\alpha}, y_{\alpha} \rightarrow_{\alpha} 0$ for the strong operator topology. It follows from Proposition 3.6 that

$$
\max \left\{\sup _{x \in B(\mathcal{M})}\left\|x_{\alpha} y_{\epsilon} x\right\|_{E}, \sup _{x \in B(\mathcal{M})}\left\|x y_{\epsilon} y_{\alpha}\right\|_{E}\right\} \leq \max \left\{\left\|x_{\alpha} y_{\epsilon}\right\|{ }_{E},\left\|y_{\epsilon} y_{\alpha}\right\|_{E}\right\} \rightarrow_{\alpha} 0,
$$

and this suffices to prove the implication (iii) $\Longrightarrow$ (iv).

Finally, the implication (iv) $\Longrightarrow(\mathrm{i})$ is clear since $e_{n} \rightarrow_{n} 0$ for the strong operator topology whenever $0 \leq e_{n} \downarrow_{n} 0 \subseteq P(\mathcal{M})$.

It should be noted that the proof of the implication (iii) $\Longrightarrow$ (iv) of the preceding theorem also holds under the assumption $y_{\epsilon} \in E^{a n}$, and this yields the following consequence.

Corollary 3.13. Let $E \subseteq S(\tau)$ be a symmetric space. If $y \in E^{a n}$, then each of the sets y $B(\mathcal{M}), B(\mathcal{M}) y$ is of uniformly absolutely continuous norm.

The preceding theorem is shown in 38 for the non-commutative Haagerup $L^{p}$ spaces associated with an arbitrary von Neumann algebra. Their method depends on the fact that the class of Haagerup $L^{p}$-spaces is closed under ultrapowers. However, it is not true that the class of $L^{p}$-spaces associated with semifinite von Neumann algebras is closed under ultrapowers [37], and so the techniques of [38] do not apply to general spaces $E$ considered here.

It is convenient to make the following definition.

Definition 3.14. If $\mathcal{A} \subseteq E$ is bounded, then $\mathcal{A}$ is said to be of right (respectively, left) uniformly absolutely continuous norm if and only if

$$
\sup \left\{\left\|x e_{n}\right\|_{E}: x \in \mathcal{A}\right\} \rightarrow_{n} 0
$$

(respectively,

$$
\left.\sup \left\{\left\|e_{n} x\right\|_{E}: x \in \mathcal{A}\right\} \rightarrow_{n} 0\right)
$$

as $n \rightarrow \infty$ for all sequences $\left\{e_{n}\right\}_{n=1}^{\infty} \subseteq P(\mathcal{M})$ for which $e_{n} \downarrow_{n} 0$.

If $\mathcal{A}$ is of both left and right uniformly absolutely continuous norm, then $\mathcal{A}$ will be said to be of $b i$-uniformly absolutely continuous norm.

Note that if $\mathcal{A}$ is of right or left uniformly absolutely continuous norm, then $\mathcal{A}$ is of uniformly absolutely continuous norm, in particular, $\mathcal{A} \subseteq E^{a n}$. It is clear that if $\mathcal{A}$ is of right (respectively, left) uniformly absolutely continuous norm, then $\mathcal{A}^{*}$ is of left (respectively, right) uniformly absolutely continuous norm. 
Proposition 3.15. Suppose that $E \subseteq S(\tau)$ is a strongly symmetrically normed space. If $\mathcal{A} \subseteq E_{+}$is bounded, then the following statements are equivalent.

(i) $\mathcal{A}$ is of uniformly absolutely continuous norm.

(ii) $\mathcal{A}$ is of right uniformly absolutely continuous norm.

(iii) $\mathcal{A}$ is of left uniformly absolutely continuous norm.

(iv) $\mathcal{A}$ is of bi-uniformly absolutely continuous norm.

Proof. Since $\|$ exe $\left\|_{E} \leq\right\| e x\left\|_{E}=\right\| x e \|_{E}$ for all $x \in \mathcal{A} \subseteq E_{+}$and all $e \in P(\mathcal{M})$, the implication (ii) $\Longrightarrow$ (i) and the equivalences (ii) $\Longleftrightarrow($ iii $) \Longleftrightarrow($ iv) are clear. The implication (i) $\Longrightarrow$ (ii) now follows by observing that

$$
\|x e\|_{E} \leq 4\left(\sup _{x \in \mathcal{A}}\|x\|_{E}^{1 / 2}\right)\|e x e\|_{E}^{1 / 2}
$$

for all $x \in \mathcal{A} \subseteq E_{+}$and all $e \in P(\mathcal{M})$, using [19], Lemma 4.4.

It will be useful to make the following observation. If $x \in E$ and if $y \in \mathcal{M}$ and if $x=u|x|$ is the polar decomposition, then

$$
\|x y\|_{E}=\|u|x| y\|_{E} \leq\||x| y\|_{E}=\left\|u^{*} x y\right\|_{E} \leq\|x y\|_{E} .
$$

In particular,

$$
\|x y\|_{E}=\||x| y\|_{E}, \quad x \in E, y \in \mathcal{M} .
$$

Corollary 3.16. Suppose that $E \subseteq S(\tau)$ is a strongly symmetrically normed space. If $\mathcal{A} \subseteq E$ is bounded and if $|\mathcal{A}|:=\{|x|: x \in \mathcal{A}\}$ is of uniformly absolutely continuous norm, then $\mathcal{A}$ is of right uniformly absolutely continuous norm.

Proof. Since $|\mathcal{A}|$ is of uniformly absolutely continuous norm, it follows from Proposition 3.15 that $|\mathcal{A}|$ is of right uniformly absolutely continuous norm. Using the above observation and noting that $\|x e\|_{E}=\|x \mid e\|_{E}$ for all $x \in \mathcal{A}, e \in P(\mathcal{M})$, the assertion of the corollary now follows readily.

Proposition 3.17. Suppose that $E \subseteq S(\tau)$ is a symmetrically normed space. If $\mathcal{A} \subseteq E$ is bounded, then the following statements are equivalent.

(i) $\mathcal{A}$ is of right (respectively, left) uniformly absolutely continuous norm.

(ii) $|\mathcal{A}|$ (respectively, $\left|\mathcal{A}^{*}\right|$ ) is of uniformly absolutely continuous norm.

Proof. To see the implication (i) $\Longrightarrow\left(\right.$ ii), suppose that $e_{n} \downarrow_{n} 0 \subseteq P(\mathcal{M})$. Using the equality $\|x e\|_{E}=\||x| e\|_{E}$ for all $x \in E, e \in P(\mathcal{M})$, observe that if $e_{n} \downarrow_{\alpha} 0 \subseteq P(\mathcal{M})$, then

$$
\sup _{x \in \mathcal{A}}\left\|e_{n}|x| e_{n}\right\|_{E} \leq \sup _{x \in \mathcal{A}}\left\||x| e_{n}\right\|_{E}=\sup _{x \in \mathcal{A}}\left\|x e_{n}\right\|_{E} \rightarrow_{n} 0,
$$

and this implies that $|\mathcal{A}|$ is of uniformly absolutely continuous norm. Since $\mathcal{A}$ is of left absolutely continuous norm if and only if $\mathcal{A}^{*}$ is of right absolutely continuous norm, the second assertion now follows from the first.

(ii) $\Longrightarrow$ (i) Note that, as observed in Corollary 3.16, the assumption that $|\mathcal{A}|$ has uniformly absolutely norm automatically implies that $|\mathcal{A}|$ is of right uniformly absolutely continuous norm and the equality $\|x e\|_{E}=\||x| e\|_{E}$ implies that $\mathcal{A}$ is of right uniformly absolutely continuous norm. If $\left|\mathcal{A}^{*}\right|$ has uniformly absolutely continuous norm, then Corollary 3.16 implies that $\left|\mathcal{A}^{*}\right|$ is of right uniformly absolutely continuous norm. Consequently $\mathcal{A}^{*}$ is of right absolutely continuous norm, and so $\mathcal{A}$ is of left absolutely continuous norm. 
In the case that $\tau(\mathbf{1})<\infty$, there are several additional characterisations of sets of uniformly absolutely continuous norm in terms of one-sided conditions. Some preliminary remarks are necessary.

If $\tau(\mathbf{1})<\infty$, then the $\sigma$-strong topology on $B(\mathcal{M})$ (and therefore the strong topology on $B(\mathcal{M})$ ) is given by the metric

$$
d(x, y)=\tau\left((x-y)^{*}(x-y)\right)^{1 / 2}=\|x-y\|_{L^{2}(\tau)}, \quad x, y \in B(\mathcal{M})
$$

(see, for example, 42, Proposition 5.3, Chapter III, p. 148). With this metric, the unit ball $B(\mathcal{M})$ is a complete metric space for the strong operator topology.

Recall further (42, Chapter V, Lemma 2.27 and Lemma 2.28, p. 326) that the von Neumann algebra $\mathcal{M}$ is finite if and only if the map $x \rightarrow x^{*}$ is $\sigma$-strongly continuous on bounded parts of $\mathcal{M}$ or, equivalently, strongly continuous on bounded parts of $\mathcal{M}$.

Lemma 3.18. Suppose that $E \subseteq S(\tau)$ is a symmetrically normed space and that $\tau(\mathbf{1})<\infty$. If $y \in E^{a n}$ and if $e_{n} \downarrow_{n} 0 \subseteq P(\mathcal{M})$, then

$$
\sup _{x \in B(\mathcal{M})}\left\|e_{n} x y\right\|_{E} \rightarrow_{n} 0 .
$$

Proof. If the assertion of the lemma does not hold, then it may be assumed that there exists $\epsilon>0$, a sequence $\left\{x_{n}\right\}_{n=1}^{\infty} \subseteq B(\mathcal{M})$ and a sequence $\left\{e_{n}\right\}_{n=1}^{\infty} \subseteq P(\mathcal{M})$ such that $e_{n} \downarrow_{n} 0 \subseteq P(\mathcal{M})$ and

$$
\left\|e_{n} x_{n} y\right\|_{E} \geq \epsilon, \quad n \in \mathbb{N} .
$$

Consequently, $x_{n}^{*} e_{n} \rightarrow_{n} 0$ for the strong operator topology, since the sequence $\left\{x_{n}\right\}_{n=1}^{\infty} \subseteq \mathcal{M}$ is bounded. By the remarks preceding the lemma, the fact that $\tau(\mathbf{1})<\infty$ now implies that $e_{n} x_{n} \rightarrow_{n} 0$ for the strong operator topology. It follows therefore that

$$
\tau\left(\left(e_{n} x_{n}\right)^{*} e_{n} x_{n}\right)=\left\|e_{n} x_{n}\right\|_{L^{2}(\tau)}^{2} \rightarrow_{n} 0,
$$

and this implies that $e_{n} x_{n} \rightarrow_{n} 0$ for the measure topology. This implies further that $e_{n} x_{n} y \rightarrow_{n} 0$ for the measure topology. On the other hand, by Corollary 3.13 . it follows that $\left\{e_{n} x_{n} y\right\}_{n=1}^{\infty} \subseteq E^{a n}$ is of uniformly absolutely continuous norm. It now follows from Theorem 3.4 that $\left\|e_{n} x_{n} y\right\|_{E} \rightarrow_{n} 0$, and this is a contradiction.

Corollary 3.19. Suppose that $\tau(\mathbf{1})<\infty$. If $y \in E^{a n}$ and if $e_{n} \downarrow_{n} 0 \subseteq P(\mathcal{M})$, then

$$
\sup _{x \in B(\mathcal{M})}\left\|y x e_{n}\right\|_{E} \rightarrow_{n} 0 .
$$

Proof. Simply observe that

$$
\left\|y x e_{n}\right\|_{E}=\left\|\left(y x e_{n}\right)^{*}\right\|_{E}=\left\|e_{n} x^{*} y^{*}\right\|_{E}
$$

for all $n \in \mathbb{N}$ and apply the preceding lemma with $y$ replaced by $y^{*}$.

Proposition 3.20. Suppose that $E \subseteq S(\tau)$ is a strongly symmetric space with order continuous norm. If $\tau(\mathbf{1})<\infty$ and if $\mathcal{A} \subseteq E$ is bounded, then the following statements are equivalent.

(i) $\mathcal{A}$ is of uniformly absolutely continuous norm.

(ii) $\mathcal{A}$ is of right uniformly absolutely continuous norm.

(iii) $\mathcal{A}$ is of left uniformly absolutely continuous norm. 
Proof. Each of the implications (ii) $\Longrightarrow(\mathrm{i}),($ iii $) \Longrightarrow(\mathrm{i})$ is clear since

$$
\left\|e_{n} x e_{n}\right\|_{E} \leq\left\|e_{n} x\right\|_{E},\left\|x e_{n}\right\|_{E}
$$

for all $n \in \mathbb{N}$. To prove the implication (i) $\Longrightarrow$ (ii), we assume that $\mathcal{A}$ is of uniformly absolutely continuous norm and let $\epsilon>0$ be given. By Theorem 3.12, there exists $y_{\epsilon} \in \mathcal{F}(\tau)$ such that

$$
\mathcal{A} \subseteq y_{\epsilon} B(\mathcal{M})+B(\mathcal{M}) y_{\epsilon}+\epsilon B(E) .
$$

Now suppose that $e_{n} \downarrow_{n} 0 \subseteq P(\mathcal{M})$. If $x \in \mathcal{A}$, then

$$
\begin{aligned}
\left\|x e_{n}\right\|_{E} & \leq \sup _{y \in B(\mathcal{M})}\left\|y y_{\epsilon} e_{n}\right\|_{E}+\sup _{y \in B(\mathcal{M})}\left\|y_{\epsilon} y e_{n}\right\|_{E}+\epsilon \\
& \leq\left\|y_{\epsilon} e_{n}\right\|_{E}+\sup _{y \in B(\mathcal{M})}\left\|y_{\epsilon} y e_{n}\right\|_{E}+\epsilon .
\end{aligned}
$$

Since $E$ has order continuous norm and $y_{\epsilon} \in \mathcal{F}(\tau)$, it follows from Theorem 3.1 that $\left\|y_{\epsilon} e_{n}\right\|_{E} \rightarrow_{n}$ 0. Using Corollary 3.19 , it follows that $\sup _{y \in B(\mathcal{M})}\left\|y_{\epsilon} y e_{n}\right\|_{E} \rightarrow_{n} 0$. It now follows that

$$
\limsup _{n} \sup _{x \in \mathcal{A}}\left\|x e_{n}\right\|_{E} \leq \epsilon,
$$

and this suffices to prove the implication (i) $\Longrightarrow$ (ii).

The proof of the implication (i) $\Longrightarrow$ (iii) is similar, and this suffices to complete the proof of the proposition.

The preceding proposition is noted in 38 in the setting of the Haagerup $L^{p}$ spaces. In the case that $\mathcal{M}$ is non-atomic, it is proved in [7] and in [26] in the special case of $L^{p}$-spaces. However, it does not seem to be the case that the general case considered here can be reduced to the non-atomic case by the usual trick of embedding $\mathcal{M}$ into $\mathcal{M} \otimes L^{\infty}[0,1]$.

Corollary 3.21. Suppose that $\tau(\mathbf{1})<\infty$ and that $E \subseteq S(\tau)$ has order continuous norm. If $\mathcal{A} \subseteq E$ the following statements are equivalent.

(i) $\mathcal{A}$ is of uniformly absolutely continuous norm.

(ii) $|\mathcal{A}|$ is of uniformly absolutely continuous norm.

(iii) $\left|\mathcal{A}^{*}\right|$ is of uniformly absolutely continuous norm.

Proof. If $x \in E$ and if $e \in P(\mathcal{M})$, then

$$
\mu(x e)=\mu(|x| e) .
$$

Since

$$
\mu(e x)=\mu\left((e x)^{*}\right)=\mu\left(x^{*} e\right)=\mu\left(\left|x^{*}\right| e\right)=\mu\left(e\left|x^{*}\right|\right),
$$

it follows that

$$
\|x e\|_{E}=\||x| e\|_{E}, \quad\|e x\|_{E}=\left\|e\left|x^{*}\right|\right\|_{E} .
$$

The equivalence (i) $\Longleftrightarrow$ (ii) now follows from the equivalence (i) $\Longleftrightarrow$ (ii) of Proposition 3.20 , while the equivalence (i) $\Longleftrightarrow$ (iii) follows from the equivalence (i) $\Longleftrightarrow$ (iii) of the same proposition.

An inspection of and simple modifications to the proof of Theorem 3.12 now yield the following. Details are omitted.

Theorem 3.22. Let $E \subseteq S(\tau)$ be a symmetric space with order continuous norm. If $\mathcal{A} \subseteq E$ is bounded, then the following statements are equivalent.

(i) $\mathcal{A}$ is of right (respectively, left) uniformly absolutely continuous norm. 
(ii) For every $\epsilon>0$, there exists $0 \leq y_{\epsilon} \in \mathcal{F}(\tau)$ such that

$$
\mathcal{A} \subseteq B(\mathcal{M}) y_{\epsilon}+\epsilon B(E) \quad \text { (respectively, } \mathcal{A} \subseteq y_{\epsilon} B(\mathcal{M})+\epsilon B(E) \text { ). }
$$

(iii) For every bounded net $\left\{x_{\alpha}\right\} \subseteq \mathcal{M}_{+}$such that $x_{\alpha} \rightarrow_{\alpha} 0$ for the strong operator topology,

$$
\left.\sup _{x \in \mathcal{A}}\left\|x x_{\alpha}\right\|_{E} \rightarrow_{\alpha} 0 \quad \text { (respectively, } \sup _{x \in \mathcal{A}}\left\|x_{\alpha} x\right\|_{E} \rightarrow_{\alpha} 0\right) \text {. }
$$

Theorem 3.23. Let $E \subseteq S(\tau)$ be a symmetric space with order continuous norm. If $\mathcal{A} \subseteq E$ is bounded, then the following statements are equivalent.

(i) $\mathcal{A}$ is of bi-uniformly absolutely continuous norm.

(ii) For every $\epsilon>0$, there exists $0 \leq y_{\epsilon} \in \mathcal{F}(\tau)$ such that

$$
\mathcal{A} \subseteq y_{\epsilon} B(\mathcal{M}) y_{\epsilon}+\epsilon B(E) .
$$

It is worth noting that the element $y_{\epsilon} \in \mathcal{F}(\tau)$ that occurs in the statements of Theorems 3.12, 3.22, and 3.23 may be taken to be a multiple of some finite trace projection, as is shown by an inspection of the proof of Theorem 3.12 .

\section{Relatively Weakly COMPaCt Subsets of $E$}

Lemma 4.1. If $E \subseteq S(\tau)$ has order continuous norm and if $0 \leq x \in E$, then each of the sets $B(\mathcal{M}) x, x B(\mathcal{M}) \subseteq E$ are weakly compact.

Proof. Let $\left\{z_{\alpha}\right\} \subseteq B(\mathcal{M})$ be any set. Since $B(\mathcal{M})$ is $\sigma\left(\mathcal{M}, L^{1}(\tau)\right)$ compact, it may be assumed that there exists $z \in B(\mathcal{M})$ with $z_{\alpha} \rightarrow_{\alpha} z$ for the weak topology $\sigma\left(\mathcal{M}, L^{1}(\tau)\right)$. It then follows that

$$
\tau\left(z_{\alpha} x y\right) \rightarrow \tau(z x y)
$$

for all $y \in E^{\times}=E^{*}$. This implies that $B(\mathcal{M}) x$ is weakly compact. Consequently, the set $x B(\mathcal{M})$ is also weakly compact, which follows from the $\sigma\left(E, E^{*}\right)=\sigma\left(E, E^{\times}\right)$ continuity of the map $x \rightarrow x^{*}, x \in E$.

Proposition 4.2. Suppose that $E \subseteq S(\tau)$ has order continuous norm. If $\mathcal{A} \subseteq E$ is of uniformly absolutely continuous norm, then $\mathcal{A}$ is relatively weakly compact.

Proof. By the implication (i) $\Longrightarrow$ (ii) of Theorem 3.12, for each $\epsilon>0$, there exists $0 \leq y_{\epsilon} \in \mathcal{F}(\tau)$ such that

$$
\mathcal{A} \subseteq B(\mathcal{M}) y_{\epsilon}+y_{\epsilon} B(\mathcal{M})+\epsilon B(E) .
$$

By the preceding lemma, each of the sets $B(\mathcal{M}) y_{\epsilon}, y_{\epsilon} B(\mathcal{M})$ are weakly compact, and from this it follows that $\mathcal{A}$ is relatively weakly compact in $E$ (see e.g. Theorem 10.17 in [1]).

The preceding proposition is given in 36, Proposition 2.7 (where the term $E$ equi-integrability was used). The present proof is, perhaps, more transparent. Via Theorem 4.4 the preceding proposition is a non-commutative counterpart to a wellknown theorem in Banach lattices which asserts that each $L$-weakly compact set in a Banach lattice with order continuous norm is weakly compact (see e.g. 11, 31]).

The converse to the preceding proposition fails in general, even in the case that $\mathcal{M}$ is commutative. Indeed, if $E=L^{p}[0, \infty), 1<p<\infty$, then bounded sets in $E$ are relatively weakly compact, since $E$ is reflexive. On the other hand, if $x_{n}=\chi_{(n, n+1]}, \quad n \in \mathbb{N}$, then the sequence $\left\{x_{n}\right\}_{n=1}^{\infty}$ is disjointly supported and 
$\left\|x_{n}\right\|_{p}=1$ for all $n \in \mathbb{N}$. This clearly implies that the sequence $\left\{x_{n}\right\}_{n=1}^{\infty}$ is not of uniformly absolutely continuous norm.

It is worth noting that a special consequence of Proposition 4.2 is that if $E \subseteq S(\tau)$ has order continuous norm, then, for every $0 \leq x \in E$, the order interval $[0, x]=$ $\{0 \leq y \in E: 0 \leq y \leq x\}$ is relatively weakly compact. The converse assertion holds as the following proposition shows. Furthermore, the next proposition answers a question in the recent preprint [35] concerning the non-commutative counterpart to well-known characterisations of Banach lattices with order continuous norm. To this end, we first need some definitions.

If $\pi: E \rightarrow E^{* *}$ is the natural embedding, then $\pi$ is a positive linear mapping. $E$ will be said to be an order ideal in $E^{* *}$ under the natural embedding if whenever $0 \leq \phi \in E^{* *}$ satisfies $0 \leq \phi \leq \pi(x)$ for some $0 \leq x \in E$, it follows that there exists $0 \leq z \in E$ such that $\phi=\pi(z)$.

Proposition 4.3. If $E \subseteq S(\tau)$ is a strongly symmetric space, then the following statements are equivalent.

(i) E has order continuous norm.

(ii) Whenever $0 \leq x \in E$ and $\left\{e_{n}\right\}_{n=1}^{\infty} \subseteq P(\mathcal{M})$ is any sequence of mutually orthogonal projections, it follows that $\left\|e_{n} x e_{n}\right\|_{E} \rightarrow_{n} 0$.

(iii) For each $0 \leq x \in E$, the order interval $[0, x]$ is $\sigma\left(E, E^{*}\right)$-compact.

(iv) $E$ is an order ideal in $E^{* *}$.

Proof. The equivalence (i) $\Longleftrightarrow($ ii $)$ follows immediately from the equivalence (vi) $\Longleftrightarrow$ (viii) of Theorem 3.1

(i) $\Longrightarrow$ (iii) Since the norm on $E$ is order continuous, it follows that any order interval $[0, x]$ is of absolutely uniformly continuous norm, and hence, by Proposition 4.2 , any order interval $[0, x]$ is relatively weakly compact. Since order intervals are norm closed and convex, we see that they are weakly closed and hence weakly compact.

(iii) $\Longrightarrow$ (i) Suppose that $0 \leq x_{\alpha} \leq x \in E(\tau)$ for all $\alpha$ and that $x_{\alpha} \downarrow_{\alpha} 0$. Since the order interval $[0, x]$ is assumed to be weakly compact, it follows that there exists $y \in[0, x]$ such that $x_{\alpha} \rightarrow_{\alpha} y$ for the $\sigma\left(E, E^{*}\right)$ topology. Since $x_{\alpha} \downarrow_{\alpha} 0$, it follows that $\phi(y)=0$ for each $0 \leq \phi \in E^{*}$. Since the dual cone in the ordered Banach space $E^{*}$ is generating (see [4]), it follows that each element of $E^{*}$ is normal and hence the norm on $E$ is order continuous.

(i) $\Longrightarrow$ (iv) Since the norm on $E$ is order continuous, it follows from [21, Chapter IV, Theorem 7.9, or [15] that $E^{*}=E^{\times}$so that $E^{* *}=E^{\times *}$. Now suppose that $0 \leq \phi \in E^{\times *}$ satisfies $0 \leq \phi \leq \pi(x)$ for some $0 \leq x \in E$. This implies that $0 \leq \phi \in E^{\times *}$ is normal and therefore $\phi \in E^{\times \times}$. Since $E$ is an order ideal in $S(\tau)$, it follows that $\phi \in E$.

(iv) $\Longrightarrow$ (iii) By the Banach-Alaoglu theorem, each order interval in $E^{* *}$ is $\sigma\left(E^{* *}, E^{*}\right)$ compact. Since $E$ is an order ideal in $E^{* *}$, it follows immediately that each order interval $[0, x], x \in E$, is $\sigma\left(E, E^{*}\right)$-compact.

As shown in 38, the criterion given in Theorem 3.12 is valid for the preduals of arbitrary von Neumann algebras and may be applied to establish the classical theorem of Akemann $[\mathrm{Ak}]$ characterising relatively weakly compact sets in von Neumann algebra preduals as precisely those bounded sets of uniformly absolutely continuous norm (see e.g. 42], Chapter III, Theorem 5.4). For convenience of 
reference, we now state the following (restricted) version of the Akemann theorem which is central to the present discussion.

Theorem 4.4 (Akemann). If $\mathcal{A} \subseteq L^{1}(\tau)$ is bounded, then the following statements are equivalent.

(i) $\mathcal{A}$ is relatively weakly compact.

(ii) $\mathcal{A}$ is of uniformly absolutely continuous norm.

Corollary 4.5. If $\tau(\mathbf{1})<\infty$ and if $\mathcal{A} \subset L^{1}(\tau)$ is bounded, then the following statements are equivalent.

(i) $\mathcal{A}$ is relatively weakly compact.

(ii)

$$
\lim _{\delta \rightarrow 0} \sup _{x \in \mathcal{A}} \omega(x ; \delta)=0 .
$$

The corollary follows immediately from Theorem 4.4 and Corollary 3.11] In the case that $\mathcal{M}$ is commutative, the preceding result is well known. In the noncommutative setting, it may be found in [26] in the case that $\mathcal{M}$ is non-atomic.

Corollary 4.6. If $\tau(\mathbf{1})<\infty$ and if $\mathcal{A} \subseteq L^{1}(\tau)$, then the following statements are equivalent.

(i) $\mathcal{A}$ is relatively weakly compact.

(ii) $|\mathcal{A}|$ is relatively weakly compact.

(iii) $\left|\mathcal{A}^{*}\right|$ is relatively weakly compact.

The corollary is an immediate consequence of the preceding theorem and Corollary 3.21 .

If $\tau(\mathbf{1})=\infty$, then neither of the implications (i) $\Longrightarrow$ (ii), (iii) of the preceding corollary is valid in general. By way of example (see [42]), let $\mathcal{H}$ be a separable Hilbert space of infinite dimension and let $\left\{\xi_{n}\right\}_{n=1}^{\infty}$ be an orthonormal basis in $\mathcal{H}$. Let $\mathcal{M}$ be $\mathcal{L}(\mathcal{H})$, equipped with the canonical trace $\tau$ given by

$$
\tau(x)=\sum_{n=1}^{\infty}\left\langle x \xi_{n}, \xi_{n}\right\rangle, \quad x \in \mathcal{L}(\mathcal{H}) .
$$

Denote by $e_{n}, n \in \mathbb{N}$, the projection onto the one-dimensional subspace spanned by $\xi_{n}$ and define the rank one partial isometry $u_{n} \in \mathcal{L}(\mathcal{H})$ by setting

$$
u_{n} \xi:=\left\langle\xi, \xi_{n}\right\rangle \xi_{1}, \quad \xi \in \mathcal{H}, \quad n \in \mathbb{N} .
$$

It is not difficult to see that $\tau\left(u_{n} x\right) \rightarrow_{n} 0$ for all $x \in \mathcal{M}$. In particular, the sequence $\left\{u_{n}\right\}_{n=1}^{\infty} \subseteq L^{1}(\tau)$ is relatively weakly compact, and so also is the sequence $\left\{u_{n}^{*}\right\}_{n=1}^{\infty} \subseteq L^{1}(\tau)$. Now observe that $\left|u_{n}\right|=e_{n}$ for all $n \in \mathbb{N}$. Since

$$
\tau\left(\left|u_{n}\right| e_{n}\right)=\tau\left(e_{n}\right)=1, \quad n \in \mathbb{N},
$$

it follows that the sequence $\left\{\left|u_{n}\right|\right\}_{n=1}^{\infty}$ is not of uniformly absolutely continuous norm and hence is not relatively weakly compact in $L^{1}(\tau)$. At the same time, if $v_{n}=u_{n}^{*}, n \in \mathbb{N}$, then the sequence $\left\{v_{n}\right\}_{n=1}^{\infty}$ is relatively weakly compact in $L^{1}(\tau)$, but the sequence $\left\{\left|v_{n}^{*}\right|\right\}_{n=1}^{\infty}=\left\{\left|u_{n}\right|\right\}_{n=1}^{\infty}$ is not relatively weakly compact. However, the implications (ii), (iii) $\Longrightarrow$ (i) of Corollary 4.6 still hold in full generality.

Corollary 4.7. If $\mathcal{A} \subseteq L^{1}(\tau)$ and if either $|\mathcal{A}|$ or $\left|\mathcal{A}^{*}\right|$ is weakly relatively compact, then $\mathcal{A}$ is weakly relatively compact. 
Proof. If $|\mathcal{A}|$ is relatively weakly compact, then it follows from the implication (i) $\Longrightarrow$ (ii) of Theorem 4.4 that $|\mathcal{A}|$ is of uniformly absolutely continuous norm. It follows from Corollary 3.16 that $\mathcal{A}$ is of uniformly absolutely continuous norm. Consequently, from the implication (ii) $\Longrightarrow$ (i) of Theorem 4.4 (or from Proposition 4.2) it follows that $\mathcal{A}$ is weakly relatively compact. Similarly, if $\left|\mathcal{A}^{*}\right|$ is weakly relatively compact, then $\mathcal{A}^{*}$ is weakly relatively compact, and hence so is $\mathcal{A}$, since involution is weakly continuous.

Finally, it should be mentioned that $\mathcal{M}$ is finite if and only if, whenever $\mathcal{A} \subseteq$ $L^{1}(\tau)$ is relatively weakly compact, it follows that $|\mathcal{A}|$ is relatively weakly compact (see e.g. [39]).

\section{Relatively COMPACT SETS in $E$}

Let us now observe that relatively compact subsets of $E$ are characterised in terms of sets of uniformly absolutely continuous norm by Theorem 3.4. Indeed, a rephrasing of this theorem shows immediately that if $E$ has order continuous norm and if $\mathcal{A} \subseteq E$, then $\mathcal{A}$ is compact if and only if $\mathcal{A}$ is compact for the measure topology and $\mathcal{A}$ is of uniformly absolutely continuous norm. The following refinement of this characterisation in the case that $\mathcal{A} \subseteq E_{+}$is given in [18, Proposition 4.6. It will be needed in the section to follow.

Proposition 5.1. Suppose that $E \subseteq S(\tau)$ is strongly symmetric. If $\mathcal{A} \subseteq E_{+}$is bounded, and if the norm on $E$ is order continuous, then the following statements are equivalent.

(i) $\mathcal{A}$ is relatively compact.

(ii) $\mathcal{A}$ has uniformly absolutely continuous norm and $h^{1 / 2} \mathcal{A} h^{1 / 2}$ is relatively compact in $L^{1}(\tau)$ for all $0 \leq h \in E^{\times}$.

To proceed further, recall (see [42, Chapter III.5) that a von Neumann algebra $\mathcal{M}$ is called atomic if every non-zero projection in $\mathcal{M}$ majorises a non-zero minimal projection. A projection in $\mathcal{M}$ is said to be of finite rank if it is the sum of finitely many orthogonal minimal projections.

The implication (ii) $\Longrightarrow$ (i) of the proposition which follows is proved in [42, Proposition III.5.10, in the special case that $E=L^{1}(\tau)$.

Proposition 5.2. Suppose that $\mathcal{M}$ is atomic and let $E \subseteq S(\tau)$ be a symmetrically normed space. If the norm on $E$ is order continuous, and if $\mathcal{A} \subseteq E$, then the following statements are equivalent.

(i) $\mathcal{A}$ is relatively compact.

(ii) Each of the sets $|\mathcal{A}|,\left|\mathcal{A}^{*}\right|$ is of uniformly absolutely continuous norm.

Proof. To see that (i) $\Longrightarrow$ (ii), it suffices to show that if $x_{n}, x \in E, n \in \mathbb{N}$, satisfies $\left\|x_{n}-x\right\|_{E} \rightarrow_{n} 0$, then each of the sequences $\left\{\left|x_{n}\right|\right\},\left\{\left|x_{n}^{*}\right|\right\}$ is of uniformly absolutely continuous norm. Suppose then that $e_{k} \downarrow_{k} 0 \subseteq P(\mathcal{M})$ and let $\epsilon>0$ be given. There exists $N \in \mathbb{N}$ such that $\left\|x_{n}-x\right\|_{E}<\epsilon$ for all $n \geq N$. It follows that

$$
\left\|x_{n} e_{k}\right\|_{E} \leq\left\|\left(x_{n}-x\right) e_{k}\right\|_{E}+\left\|x e_{k}\right\|_{E} \leq \epsilon+\left\|x e_{k}\right\|_{E}, \quad n \geq \mathbb{N} .
$$

Using this estimate and the fact that the norm on $E$ is order continuous, it follows that $\sup _{n \geq N}\left\|x_{n} e_{k}\right\|_{E} \rightarrow_{k} 0$. Again, order continuity of the norm on $E$ implies that 
$\sup _{1 \leq n \leq N}\left\|x_{n} e_{k}\right\|_{E} \rightarrow_{k} 0$ and so

$$
\sup _{n \in \mathbb{N}}\left\|x_{n} e_{k}\right\|_{E} \rightarrow_{k} 0 \text {. }
$$

This implies further that

$$
\sup \left\{\left\|w x_{n} e_{k}\right\|_{E}: w \in B(\mathcal{M}), n \in \mathbb{N}\right\} \leq \sup _{n \in \mathbb{N}}\left\|x_{n} e_{k}\right\|_{E} \rightarrow_{\alpha} 0 .
$$

Using the polar decomposition, it now follows that

$$
\sup _{n \in \mathbb{N}}\left\|\left|x_{n}\right| e_{k}\right\|_{E} \rightarrow_{k} 0 .
$$

By Proposition 3.15, it follows that $\left\{\left|x_{n}\right|\right\} \subseteq E_{+}$has uniformly absolutely continuous norm. Since $\left\|x_{n}^{*}-x^{*}\right\|_{E} \rightarrow 0$, the same argument now shows that $\left\{\left|x_{n}^{*}\right|\right\}$ has uniformly absolutely continuous norm.

(ii) $\Longrightarrow$ (i) The assumption that $|\mathcal{A}|$ has uniformly absolutely continuous norm implies that $\mathcal{A}$ has uniformly absolutely continuous norm, by Corollary 3.16. Consequently, Proposition 4.2 implies that $\mathcal{A}$ is weakly relatively compact. Using the Eberlein-Smulian theorem, it will suffice to show that if $\left\{x_{n}\right\} \subseteq \mathcal{A}$ is weakly convergent to some $x \in E$, then the sequence $\left\{x_{n}\right\}$ is a Cauchy sequence in $E$. To this end, let $\left\{f_{\alpha}\right\} \subseteq P(\mathcal{M})$ be the system of all projections of finite rank and note that $f_{\alpha} \uparrow \mathbf{1}$, since $\mathcal{M}$ is atomic. In particular $\left(\mathbf{1}-f_{\alpha}\right) \downarrow_{\alpha} 0$. Observe that

$$
\begin{aligned}
\left\|x_{n}-x_{m}\right\|_{E} \leq & \left\|f_{\alpha}\left(x_{n}-x_{m}\right) f_{\alpha}\right\|_{E}+\left\|f_{\alpha}\left(x_{n}-x_{m}\right)\left(\mathbf{1}-f_{\alpha}\right)\right\|_{E} \\
& +\left\|\left(\mathbf{1}-f_{\alpha}\right)\left(x_{n}-x_{m}\right)\right\|_{E} \\
\leq & \left\|f_{\alpha}\left(x_{n}-x_{m}\right) f_{\alpha}\right\|_{E}+\left\|\left(x_{n}-x_{m}\right)\left(\mathbf{1}-f_{\alpha}\right)\right\|_{E} \\
& +\left\|\left(x_{n}^{*}-x_{m}^{*}\right)\left(\mathbf{1}-f_{\alpha}\right)\right\|_{E}
\end{aligned}
$$

for all $m, n \in \mathbb{N}$ and indices $\alpha$. Observe further that, for all $n, m \in \mathbb{N}$,

$$
\begin{aligned}
\left\|\left(x_{n}-x_{m}\right)\left(\mathbf{1}-f_{\alpha}\right)\right\|_{E} & \leq\left\|x_{n}\left(\mathbf{1}-f_{\alpha}\right)\right\|_{E}+\left\|x_{m}\left(\mathbf{1}-f_{\alpha}\right)\right\|_{E} \\
& =\left\|\left|x_{n}\right|\left(\mathbf{1}-f_{\alpha}\right)\right\|_{E}+\left\|\left|x_{m}\right|\left(\mathbf{1}-f_{\alpha}\right)\right\|_{E} \\
& \leq 2 \sup _{n \in \mathbb{N}}\left\|\left|x_{n}\right|\left(\mathbf{1}-f_{\alpha}\right)\right\|_{E}
\end{aligned}
$$

and similarly

$$
\left\|\left(x_{n}^{*}-x_{m}^{*}\right)\left(\mathbf{1}-f_{\alpha}\right)\right\|_{E} \leq 2 \sup _{n \in \mathbb{N}}\left\|\left|x_{n}^{*}\right|\left(\mathbf{1}-f_{\alpha}\right)\right\|_{E} .
$$

Since each of the sets $|\mathcal{A}|,\left|\mathcal{A}^{*}\right|$ has uniformly absolutely continuous norm, using Proposition 3.15, it follows that

$$
\sup _{x \in \mathcal{A}}\left\||x|\left(\mathbf{1}-f_{\alpha}\right)\right\|_{E}, \sup _{x \in \mathcal{A}}\left\|\left|x^{*}\right|\left(\mathbf{1}-f_{\alpha}\right)\right\|_{E} \rightarrow_{\alpha} 0 .
$$

In particular,

$$
\sup _{n} \max \left\{\left\|\left|x_{n}\right|\left(\mathbf{1}-f_{\alpha}\right)\right\|_{E},\left\|\left|x_{n}^{*}\right|\left(\mathbf{1}-f_{\alpha}\right)\right\|_{E}\right\} \rightarrow_{\alpha} 0 .
$$

Now suppose that $\epsilon>0$ is given, and suppose that $e=\mathbf{1}-f_{\alpha_{0}}$ satisfies

$$
\sup _{n} \max \left\{\left\|\left|x_{n}\right| e\right\|_{E},\left\|\left|x_{n}^{*}\right| e\right\|_{E}\right\}<\epsilon / 4
$$

so that

$$
\left\|x_{n}-x_{m}\right\|_{E} \leq\left\|e\left(x_{n}-x_{m}\right) e\right\|_{E}+\epsilon, \quad n, m \in \mathbb{N} .
$$


Since $e$ is a finite-rank projection, it follows that the algebra $e \mathcal{M} e$ is finite-dimensional. This implies that all linear space topologies on $e E e$ coincide. It follows that there exists $N \in \mathbb{N}$ such that

$$
\left\|e\left(x_{n}-x_{m}\right) e\right\|_{E}<\epsilon, \quad n, m \geq N .
$$

Consequently the sequence $\left\{x_{n}\right\}$ is a Cauchy sequence in $E$, and this suffices to conclude the proof of the proposition.

Corollary 5.3. Suppose that $\mathcal{M}$ is atomic and let $E \subseteq S(\tau)$ be a symmetrically normed space. If the norm on $E$ is order continuous, if $\tau(\mathbf{1})<\infty$ and if $\mathcal{A} \subseteq E$, then the following statements are equivalent.

(i) $\mathcal{A}$ is relatively compact.

(ii) $\mathcal{A}$ is of uniformly absolutely continuous norm.

The corollary follows immediately from Proposition 5.2 and Corollary 3.21

The following consequences are of particular interest.

Corollary 5.4. Suppose that $\mathcal{M}$ is atomic. If $\mathcal{A} \subseteq L_{1}(\tau)$, then the following statements are equivalent.

(i) $\mathcal{A}$ is relatively compact.

(ii) Each of the sets $|\mathcal{A}|,\left|\mathcal{A}^{*}\right|$ is weakly relatively compact.

The corollary is an immediate consequence of Proposition 5.2 via Theorem 4.4

Corollary 5.5. Suppose that $\mathcal{M}$ is atomic. If $\tau(\mathbf{1})<\infty$, and if $\mathcal{A} \subseteq L_{1}(\tau)$, then the following statements are equivalent.

(i) $\mathcal{A}$ is relatively compact.

(ii) $\mathcal{A}$ is weakly relatively compact.

The corollary follows directly from Corollary 5.3. Theorem 4.4 and Corollary 3.21

Recall that a Banach space is said to have the Schur property if and only if each weakly convergent sequence is norm convergent. Corollary [5.5] implies the classical result that the sequence space $l^{1}(\mathbb{N})$ has the Schur property. Indeed, let $\mathcal{M}$ be $l^{\infty}(\mathbb{N})$, equipped with the trace $\tau$ given by

$$
\tau(x)=\sum_{k=1}^{\infty} 2^{-k} x_{k}, \quad x=\left\{x_{k}\right\}_{k=1}^{\infty} \in l^{\infty}(\mathbb{N}) .
$$

In particular, $\tau(\mathbf{1})<\infty$. It is clear that

$$
x=\left\{x_{k}\right\}_{k=1}^{\infty} \in l^{1}(\mathbb{N}) \Longleftrightarrow \tilde{x}=\left\{2^{k} x_{k}\right\}_{k=1}^{\infty} \in L^{1}(\tau),
$$

in which case

$$
\|x\|_{l^{1}(\mathbb{N})}=\|\tilde{x}\|_{L^{1}(\tau)} .
$$

As is easily checked, $x_{n} \rightarrow x$ weakly in $l^{1}(\mathbb{N})$ if and only if $\tilde{x}_{n} \rightarrow \tilde{x}$ weakly in $L^{1}(\tau)$.

On the other hand, if $\mathcal{M}=B(\mathcal{H})$ is equipped with standard trace, then $L_{1}(\tau)$ does not have the Schur property. Indeed, if $\left\{u_{n}\right\}_{n \in \mathbb{N}}$ is the sequence of rank one partial isometries discussed in the remarks following Corollary 4.6, then sequence $\left\{u_{n}^{*}\right\}$ converges weakly to 0 in $L^{1}(\tau)$. However, for all $n \in \mathbb{N},\left|u_{n}^{*}\right|$ is the rank one projection $\xi \rightarrow\left\langle\xi, \xi_{1}\right\rangle \xi_{1}$. Consequently, $\left\|u_{n}^{*}\right\|_{L^{1}(\tau)}=1$, for all $n \in \mathbb{N}$ (see e.g. [42, Chapter III.5). 


\section{WEAK CONVERGENCE, CONVERGENCE IN MEASURE AND SETS OF UNIFORMLY ABSOLUTELY CONTINUOUS NORM}

The following submajorisation will be needed in the sequel (see also [5], 6]).

Lemma 6.1. If $e_{1}, e_{2}, \ldots, e_{n} \in \mathcal{M}$ are projections with $e_{i} e_{j}=0, i \neq j$, and if $x \in S(\tau)$, then

$$
e_{1} x e_{1}+e_{2} x e_{2}+\ldots+e_{n} x e_{n} \prec \prec x .
$$

Proof. If $\epsilon=\left(\epsilon_{1}, \epsilon_{2}, \ldots, \epsilon_{n}\right), \epsilon_{i}= \pm 1,1 \leq i \leq n$ and if $v_{\epsilon}=\sum_{i=1}^{n} \epsilon_{i} e_{i}$, then

$$
\sum_{i=1}^{n} e_{i} x e_{i}=\frac{1}{2^{n}} \sum_{\epsilon} v_{\epsilon} x v_{\epsilon} .
$$

Since $\mu(y+z) \prec \prec \mu(y)+\mu(z)$ (see e.g. [23]), for all $y, z \in S(\tau)$, it follows that

$$
\mu\left(\sum_{i=1}^{n} e_{i} x e_{i}\right) \prec \prec \frac{1}{2^{n}} \sum_{\epsilon} \mu\left(v_{\epsilon} x v_{\epsilon}\right) .
$$

Since $e_{i} e_{j}=0, i \neq j$, observe that

$$
v_{\epsilon}^{*} v_{\epsilon}=e_{1}+e_{2}+\ldots+e_{n}
$$

so that $v_{\epsilon} \in \mathcal{M}$ is a partial isometry for all $\epsilon$. Consequently

$$
\mu\left(v_{\epsilon} x v_{\epsilon}\right) \leq \mu(x)
$$

for all $\epsilon$, and the assertion of the lemma follows.

In the following, some further terminology will be useful. As usual $E \subseteq S(\tau)$ denotes a strongly symmetric space.

It will be convenient to say that a sequence $\left\{x_{n}\right\}_{n=1}^{\infty} \subseteq E$ is disjointly supported if there exists a sequence $\left\{e_{n}\right\}_{n=1}^{\infty} \subseteq P(\mathcal{M})$ of mutually orthogonal projections such that $x_{n}=e_{n} x_{n} e_{n}$ for all $n \in \mathbb{N}$.

If $X \subseteq E$ is a closed subspace, then $X$ is said to be a copy of $c_{0}$ if and only if there exists a (Banach space) isomorphism $T: c_{0} \rightarrow X$ of $c_{0}$ onto $X$. If the isomorphism $T: Y \rightarrow X$ satisfies $T z \geq 0$ whenever $0 \leq z \in c_{0}$, then $T$ will be called a positive isomorphism and the range $X$ will be termed a positive copy of $c_{0}$. If $T$ is a positive isomorphism and maps the unit vector basis of $c_{0}$ onto a disjointly supported sequence in $X$, then $X$ will be called a disjointly supported positive copy of $c_{0}$.

Lemma 6.2. Let $E \subseteq S(\tau)$ be a strongly symmetric space and suppose that $E$ has order continuous norm. Suppose that $0 \leq y \in E^{\times \times}$and that $\mathcal{A} \subseteq E$ satisfies $0 \leq x \leq y$ for all $x \in \mathcal{A}$. If $\mathcal{A}$ is not of uniformly absolutely continuous norm in $E$, then $E$ contains a positive disjointly supported positive copy of $c_{0}$.

Proof. Since $\mathcal{A}$ is not of uniformly absolutely continuous norm in $E$, it follows from Proposition 3.2 that there exists $\epsilon>0$, a sequence $0 \leq x_{n}, n \in \mathbb{N}$, in $\mathcal{A}$ and a sequence $\left\{e_{n}\right\}_{n=1}^{\infty} \subseteq P(\mathcal{M})$ of mutually disjoint projections such that

$$
\left\|e_{n} x_{n} e_{n}\right\|_{E} \geq \epsilon, \quad n \in \mathbb{N} .
$$

Denote by $\left\{q_{n}\right\}_{n=1}^{\infty} \subseteq c_{0}$ the standard vector basis in $c_{0}$. The linear mapping $T: c_{00} \rightarrow E$ is defined by setting

$$
T z=\sum_{j=1}^{k} c_{j} e_{j} x_{j} e_{j}
$$


if $z=\sum_{j=1}^{k} c_{j} q_{j} \in c_{00},\left\{c_{j}\right\}_{j=1}^{k} \subset \mathbb{R}$. If, in addition, such $z$ is positive, then, for every $j \in \mathbb{N}$, we have by Lemma 6.1

$$
\begin{aligned}
c_{j} e_{j} x_{j} e_{j} \leq T z & \leq \sum_{j=1}^{k} c_{j} e_{j} y e_{j} \\
& \leq\|z\|_{\infty} \sum_{j=1}^{k} e_{j} y e_{j} \prec \prec\|z\|_{\infty} y .
\end{aligned}
$$

Since $E^{\times \times}$is strongly symmetric and order continuity of the norm on $E$ implies that the embedding of $E$ into $E^{\times \times}$is an isometry (see [15]), it follows that

$$
\epsilon\|z\|_{\infty} \leq\|T z\|_{E} \leq\|z\|_{\infty}\|y\|_{E^{\times \times}} .
$$

As is easily verified, $|T z|=T|z|$ for all $z \in c_{00}$, and it now follows that $T$ extends uniquely to a positive isomorphism of $c_{0}$ onto the closed linear span of the sequence $\left\{e_{n} x e_{n}\right\}_{n \in \mathbb{N}}$. This suffices to complete the proof of the implication.

Using the equivalence $(\mathrm{i}) \Longleftrightarrow$ (viii) of Theorem 3.1 an inspection of the proof of the preceding lemma yields the following.

Lemma 6.3. Let $E \subseteq S(\tau)$ be strongly symmetric. If $0 \leq x \in E$ and if $x \notin E^{a n}$, then there exists a mutually orthogonal sequence of projections $\left\{e_{n}\right\}_{n=1}^{\infty}$ such that the sequence $\left\{e_{n} x e_{n}\right\}_{n=1}^{\infty}$ spans a positive disjointly supported copy of $c_{0}$.

The equivalence $(\mathrm{i}) \Longleftrightarrow(\mathrm{v})$ of Theorem 3.1 now implies the following.

Corollary 6.4. Let $E \subseteq S(\tau)$ be strongly symmetric. If $E$ contains no disjointly supported positive copy of $c_{0}$, then the norm on $E$ is order continuous.

Recall that a strongly symmetric space $E$ is said to be a KB-space if $E$ has order continuous norm and the Fatou property.

Theorem 6.5. Let $E \subseteq S(\tau)$ be strongly symmetric. The following statements are equivalent.

(i) $E$ is a KB-space.

(ii) $E$ is weakly sequentially complete.

(iii) E contains no isomorphic copy of $c_{0}$.

(iv) $E$ contains no positive isomorphic copy of $c_{0}$.

(v) E contains no disjointly supported positive copy of $c_{0}$.

Proof. The equivalences (i) $\Longleftrightarrow$ (ii) $\Longleftrightarrow$ (iii) are established in [14. The implications (iii) $\Longrightarrow($ iv $) \Longrightarrow(\mathrm{v})$ are trivial.

The proof of the theorem will be complete by showing the implication $(\mathrm{v}) \Longrightarrow(\mathrm{i})$. To this end, observe first that since $E$ contains no disjointly supported positive isomorphic copy of $c_{0}$, it follows from Corollary 6.4 that the norm on $E$ is order continuous. Now suppose that $0 \leq x_{n} \uparrow_{n} \subseteq E$ is norm-bounded. It will suffice to show that the sequence $\left\{x_{n}\right\}_{n=1}^{\infty}$ is relatively compact. Note first that $x^{\times \times}=\sup _{n} x_{n}$ exists in $E^{\times \times}$since $E^{\times \times}$has the Fatou property and $E$ is isometrically embedded into $E^{\times \times}$ due to the order continuity of the norm $E$ (see [15]). By Lemma 6.2, the assumption that $E$ contains no disjointly supported positive isomorphic copy of $c_{0}$ and the fact that $E$ has order continuous norm imply that the sequence $\left\{x_{n}\right\}_{n=1}^{\infty}$ is of uniformly absolutely continuous norm. Now suppose that $0 \leq h \in E^{\times}$. Since $0 \leq x_{n} \uparrow x^{\times \times}$ holds in $E^{\times \times}$, it follows (see [15]) that $0 \leq h^{1 / 2} x_{n} h^{1 / 2} \uparrow_{n} h^{1 / 2} x^{\times \times} h^{1 / 2}$ in $L^{1}(\tau)$. By 
order continuity of the norm on $L^{1}(\tau)$, it follows that $h^{1 / 2} x_{n} h^{1 / 2} \rightarrow_{n} h^{1 / 2} x^{\times \times} h^{1 / 2}$ in $L^{1}(\tau)$, and this implies that $\left\{h^{1 / 2} x_{n} h^{1 / 2}\right\}$ is relatively compact in $L^{1}(\tau)$. It now follows from Proposition 5.1 that the sequence $\left\{x_{n}\right\}_{n=1}^{\infty}$ is relatively compact in $E$, and this completes the proof of the theorem.

It will be convenient to adopt the following terminology. If $\mathcal{A} \subseteq S(\tau)$, then $\mathcal{A}$ will be called solid if $B(\mathcal{M}) \mathcal{A} B(\mathcal{M}) \subseteq \mathcal{A}$.

Proposition 6.6. Suppose that $E \subseteq S(\tau)$ is strongly symmetric and that $\tau(\mathbf{1})<$ $\infty$. If $E$ has order continuous norm, and if $\mathcal{A} \subseteq E$ is bounded and solid, then the following statements are equivalent.

(i) $\mathcal{A}$ is of uniformly absolutely continuous norm.

(ii) $\mathcal{A}$ is weakly relatively compact, and the norm and measure topologies coincide on $\mathcal{A}$.

Proof. (i) $\Longrightarrow$ (ii) Suppose that $\mathcal{A}$ is of uniformly absolutely continuous norm. It follows from Proposition 4.2 that $\mathcal{A}$ is weakly relatively compact. That the norm and measure topologies coincide on $\mathcal{A}$ now follows from Theorem 3.4 .

(ii) $\Longrightarrow$ (i) Now assume that $\mathcal{A}$ is relatively weakly compact and the norm and measure topologies coincide on $\mathcal{A}$.

Suppose that $\mathcal{A}$ is not of uniformly absolutely continuous norm. Then, by Definition 3.3 and Proposition 3.2 (ii), there exist a sequence $\left\{x_{n}\right\}_{n=1}^{\infty} \subseteq \mathcal{A}$ and a sequence $\left\{e_{n}\right\}_{n=1}^{\infty} \subseteq P(\mathcal{M})$ of mutually orthogonal projections, and there exists $\epsilon>0$ such that

$$
\left\|e_{n} x_{n} e_{n}\right\|_{E} \geq \epsilon, \quad n \in \mathbb{N} .
$$

Since $\tau(\mathbf{1})<\infty$, it follows that $e_{n} x_{n} e_{n} \rightarrow_{n} 0$ for the measure topology. Since $E$ has order continuous norm, it follows that $E^{*}=E^{\times}$and so the sequence $\left\{x_{n}\right\}_{n=1}^{\infty}$ is relatively $\sigma\left(E^{\times \times}, E^{\times}\right)$compact.

Since the assumption that $\tau(\mathbf{1})<\infty$ guarantees that $E^{\times}, E^{\times \times} \subseteq S_{0}(\tau)$, it follows from the implication (i) $\Longrightarrow(\mathrm{v})$ of [14], Proposition 2.8, that

$$
\sup _{n \in \mathbb{N}} \int_{[0, \infty)} \mu\left(x_{n}\right) \mu\left(y p_{n}\right) d m \rightarrow_{n} 0
$$

for every $0 \leq y \in E^{\times}$and for every mutually orthogonal sequence $\left\{p_{n}\right\}_{n=1}^{\infty} \subseteq$ $P(\mathcal{M})$. Since $\mu\left(e_{n} x_{n} e_{n}\right) \leq \mu\left(x_{n}\right), n \in \mathbb{N}$, it follows that

$$
\sup _{n \in \mathbb{N}} \int_{[0, \infty)} \mu\left(e_{n} x_{n} e_{n}\right) \mu\left(y p_{n}\right) d m \rightarrow_{n} 0
$$

for every $0 \leq y \in E^{\times}$and for every mutually orthogonal sequence $\left\{p_{n}\right\}_{n=1}^{\infty} \subseteq$ $P(\mathcal{M})$. It now follows from the implication $(\mathrm{v}) \Longrightarrow(\mathrm{i})$ of the same proposition that the sequence $\left\{e_{n} x_{n} e_{n}\right\}_{n=1}^{\infty}$ is relatively $\sigma\left(E^{\times \times}, E^{\times}\right)$-compact. It may be assumed that there exists $x \in E^{\times \times}$such that $e_{n} x_{n} e_{n} \rightarrow_{n} x$ for the weak topology $\sigma\left(E^{\times \times}, E^{\times}\right)$. It follows from [34], Lemma 5.3, that $x=0$ and so $e_{n} x_{n} e_{n} \rightarrow_{n} 0$ for the weak topology $\sigma\left(E, E^{\times}\right)$and so also for the weak topology $\sigma\left(E, E^{*}\right)$, since $E$ has order continuous norm. Since the norm and measure topologies coincide on $\mathcal{A}$, and since $\mathcal{A}$ is solid, it follows that $\left\|e_{n} x_{n} e_{n}\right\|_{E} \rightarrow_{n} 0$. This is clearly a contradiction, and so the sequence $\left\{x_{n}\right\}_{n=1}^{\infty}$ has uniformly absolutely continuous norm, and this completes the proof. 
The preceding Proposition 6.6 is modelled on 31, Proposition 3.6.7, in the Banach lattice setting. It would be interesting to know if the assumption that $E$ has order continuous norm can be omitted.

Proposition 6.7. Suppose that $E \subseteq S(\tau)$ is strongly symmetric and that $\tau(\mathbf{1})<$ $\infty$. The following statements are equivalent.

(i) Each relatively weakly compact subset of $E$ is of uniformly absolutely continuous norm.

(ii) Each two-sided disjointly supported weakly null sequence in $E$ is norm convergent to 0 .

Proof. (i) $\Longrightarrow$ (ii) Suppose that $\left\{x_{n}\right\}_{n=1}^{\infty} \subseteq E$ is two-sided disjointly supported and weakly null. Since $\tau(\mathbf{1})<\infty$, it follows that $x_{n} \rightarrow_{n} 0$ for the measure topology. Since $\left\{x_{n}\right\}_{n=1}^{\infty}$ is relatively weakly compact, it follows from the assumption of (i) that $\left\{x_{n}\right\}_{n=1}^{\infty}$ has uniformly absolutely continuous norm. In particular, $\left\{x_{n}\right\}_{n=1}^{\infty} \subseteq$ $E^{a n}$. By Theorem 3.4, it follows that $\left\|x_{n}\right\|_{E} \rightarrow_{n} 0$.

(ii) $\Longrightarrow$ (i) Observe first that the condition (ii) implies that $E$ is a KB-space. If this is not the case, then, by Theorem 6.5. there exists a two-sided disjointly supported sequence $\left\{x_{n}\right\}_{n=1}^{\infty} \subseteq E_{+}$equivalent to the unit vector basis of $c_{0}$. Such a sequence is weakly null but not norm-convergent to 0 , contradicting the assumption of (ii). Now suppose that $\mathcal{A} \subseteq E$ is relatively weakly compact but is not of uniformly absolutely continuous norm. Since $E$ is a KB-space, the norm on $E$ is order continuous. By Theorem 3.12, there exist $\epsilon>0$, a sequence $\left\{x_{n}\right\}_{n=1}^{\infty} \subseteq \mathcal{A}$ and a sequence $\left\{e_{n}\right\}_{n=1}^{\infty} \subseteq P(\mathcal{M})$ of mutually orthogonal projections such that

$$
\left\|e_{n} x_{n} e_{n}\right\|_{E} \geq \epsilon, \quad n \in \mathbb{N} .
$$

Since $\tau(\mathbf{1})<\infty$, it follows that $e_{n} x_{n} e_{n} \rightarrow_{n} 0$ for the measure topology. As in the proof of Proposition 6.6 preceding, it follows that $e_{n} x_{n} e_{n} \rightarrow_{n} 0$ weakly in $E$. The assumption of (ii) now implies that $\left\|e_{n} x_{n} e_{n}\right\|_{E} \rightarrow_{n} 0$, and this is a contradiction. This suffices to complete the proof of the proposition.

Proposition 6.7 is based on [31, Corollary 3.6.8. In view of Propositions 6.7 and 4.2, under the assumptions of Proposition 6.7, the class of all relatively weakly compact sets and the class of all sets with uniformly absolutely continuous norm coincide.

It is now convenient to introduce the following definition.

Definition 6.8. If $E \subseteq S(\tau)$, then $E$ is said to have property ( $W m$ ) if and only if, for all sequences $\left\{x_{n}\right\}_{n=1}^{\infty} \subseteq E$, whenever $x_{n} \rightarrow_{n} 0$ both weakly and in measure, it follows that $\left\|x_{n}\right\|_{E} \rightarrow_{n} 0$.

This notion was introduced in [29] (see also [3]). Detailed proofs of the results announced in [29] may be found in the unpublished manuscript 30].

To fix ideas, observe first that the space $L_{1}(\tau)$ has property $(W m)$. Indeed, any relatively weakly compact subset in $L_{1}(\tau)$ has uniformly absolutely continuous norm, as follows from Theorem 4.4, and by Theorem 3.4, any sequence in $L_{1}(\tau)$ with uniformly absolutely continuous norm which converges to 0 for the measure topology necessarily converges in norm, since the norm on $L_{1}(\tau)$ is order continuous. 
Lemma 6.9. Suppose that $\tau(\mathbf{1})<\infty$ and that $E \subseteq S(\tau)$ is strongly symmetric.

(i) If $E$ has the property $(W m)$, then $E$ is a KB-space.

(ii) If each relatively weakly compact subset of $E$ is of uniformly absolutely continuous norm, then $E$ is a KB-space.

Proof. (i) If $E$ is not a KB-space, then by Theorem 6.5 there exists a disjointly supported sequence $0 \leq x_{n} \in E$, and there exists a positive isomorphism $0 \leq$ $T: c_{0} \rightarrow \overline{\operatorname{span}}\left\{x_{n}\right\}_{n=1}^{\infty}$ such that $T q_{n}=x_{n}, n \in \mathbb{N}$. Here $\left\{q_{n}\right\}_{n \in \mathbb{N}}$ denotes the unit vector basis in $c_{0}$. In particular, it follows that there exists $\epsilon>0$ such that $\left\|x_{n}\right\|_{E} \geq \epsilon$, for all $n \in \mathbb{N}$. On the other hand, since $\tau(\mathbf{1})<\infty$, it follows that $x_{n} \rightarrow_{n} 0$ for the measure topology. Suppose that $\varphi \in E^{*}$; then, denoting by $\varphi^{\prime}$ the restriction of $\varphi$ to $\overline{\operatorname{span}}\left\{x_{n}\right\}_{n=1}^{\infty} \subseteq E$, it is clear that

$$
\begin{aligned}
\left\langle x_{n}, \varphi\right\rangle & =\left\langle x_{n}, \varphi^{\prime}\right\rangle \\
& =\left\langle T q_{n}, \varphi^{\prime}\right\rangle=\left\langle q_{n}, T^{*} \varphi^{\prime}\right\rangle .
\end{aligned}
$$

Since $T^{*} \varphi^{\prime} \in l^{1}$ and $q_{n} \rightarrow_{n} 0$ for the weak topology $\sigma\left(c_{0}, l^{1}\right)$, it follows that $\left\langle x_{n}, \varphi\right\rangle \rightarrow_{n} 0$, so that $x_{n} \rightarrow_{n} 0$ for the weak topology $\sigma\left(E, E^{*}\right)$. Since $E$ has property $(W m)$, it follows that $\left\|x_{n}\right\|_{E} \rightarrow_{n} 0$. This is clearly a contradiction and so suffices to establish the assertion of (i).

(ii) If $E$ is not a KB-space, then by Theorem 6.5, $E$ contains a disjointly supported positive copy of $c_{0}$. Let $\left\{x_{n}\right\}_{n=1}^{\infty}$ be a sequence in $E$ corresponding to the unit vector basis of $c_{0}$. Consequently, the sequence $\left\{x_{n}\right\}_{n=1}^{\infty}$ is weakly null but not norm convergent to 0 . Being weakly null, the sequence $\left\{x_{n}\right\}_{n=1}^{\infty}$ is of uniformly absolutely continuous norm by hypothesis and converges to 0 for the measure topology, since $\tau(\mathbf{1})<\infty$. By Theorem 3.4, the sequence $\left\|x_{n}\right\|_{E} \rightarrow_{n} 0$, and this is a contradiction.

Via Corollary 3.11 the proposition which follows may be found in 3], Theorem 5.5 , in the special case that $\mathcal{M}=L^{\infty}[0,1]$ with trace given by Lebesgue integral.

Proposition 6.10. Suppose that $E \subseteq S(\tau)$ is strongly symmetric. If $\tau(\mathbf{1})<\infty$, then the following statements are equivalent.

(i) E has property $(W m)$.

(ii) Each relatively weakly compact set in $E$ is of uniformly absolutely continuous norm.

Proof. Note that both conditions (i), (ii), together with the assumption that $\tau(\mathbf{1})<$ $\infty$, imply that $E$ is a KB-space, by Lemma 6.9. In particular, the norm on $E$ is order continuous.

(ii) $\Longrightarrow$ (i) Suppose that $\left\{x_{n}\right\}_{n=1}^{\infty} \subseteq E$ satisfies $x_{n} \rightarrow_{n} 0$ both weakly and in measure. The assertion of (ii) implies that $\left\{x_{n}\right\}_{n=1}^{\infty}$ has uniformly absolutely continuous norm. It then follows from Theorem 3.4 that $\left\|x_{n}\right\|_{E} \rightarrow_{n} 0$.

(i) $\Longrightarrow$ (ii) Let $\left\{x_{n}\right\}_{n=1}^{\infty} \subseteq E$ be a two-sided disjointly supported weakly null sequence. By the implication (ii) $\Longrightarrow$ (i) of Proposition 6.7 it will suffice to show that $\left\|x_{n}\right\|_{E} \rightarrow_{n} 0$. Since $\tau(\mathbf{1})<\infty$, it follows that $x_{n} \rightarrow_{n} 0$ in measure since $\left\{x_{n}\right\}_{n=1}^{\infty}$ is two-sided disjointly supported. Since $x_{n} \rightarrow_{n} 0$ weakly, the assumption of (i) implies that $\left\|x_{n}\right\|_{E} \rightarrow_{n} 0$, which completes the proof.

Let $\phi$ denote an increasing concave function on $[0, \tau(\mathbf{1}))$ for which $\phi(0)=0=$ $\phi(0+)$. Note that $\phi$ is absolutely continuous and $\phi(t)=\int_{[0, t)} \phi^{\prime}(s) d s, t>0$. Recall 
that the Lorentz space $\left(\Lambda_{\phi},\|\cdot\|_{\Lambda_{\phi}}\right)$ consists of all $x \in S(\tau)$ for which

$$
\|x\|_{\Lambda_{\phi}}=\int_{[0, \tau(\mathbf{1}))} \mu(x) d \phi<\infty .
$$

Note that if $\tau(\mathbf{1})<\infty$, then the norm on $\Lambda_{\phi}$ is order continuous. Indeed, if $x_{n} \downarrow_{n} 0 \subseteq \Lambda_{\phi}$, then $\mu\left(x_{n}\right) \downarrow_{n} 0$, and this implies that

$$
\left\|x_{n}\right\|_{\Lambda_{\phi}}=\int_{[0, \tau(\mathbf{1}))} \mu\left(x_{n}\right) \phi^{\prime} d m \downarrow_{n} 0 .
$$

If $\tau(\mathbf{1})=\infty$, then the norm on $\Lambda_{\phi}$ is order continuous if and only if $\lim _{t \rightarrow \infty} \phi(t)=$ $\infty$. The Köthe dual of the Lorentz space $\Lambda_{\phi}$ is the Marcinkiewicz space $M_{\psi}$, $\psi(t)=t / \psi(t)$ consisting of all $x \in S(\tau)$ such that

$$
\|x\|_{M_{\psi}}=\sup _{0<h<\infty}[\psi(h)]^{-1} \int_{[0, h)} \mu(x) d m<\infty
$$

(see e.g. 28, Chapter II.5).

It is the case that any non-commutative Lorentz space $\Lambda_{\phi}(\tau)$ corresponding to a separable Lorentz space on the semiaxis has property $(W m)$. This result is due to Krygin, Sheremet'ev and Sukochev [29]. Their proof (see [30]) is based on showing first the general lifting theorem that if $E \subseteq S(m)$ is a separable symmetric space and if $E$ has property $(W m)$, then $E(\tau)$ has property $(W m)$ for every semifinite $(\mathcal{M}, \tau)$ and then using results in [8] and [44. We will present here an independent and more direct proof which does not depend on [30, which is not generally available. A proof of the lifting theorem will be indicated in the following section for the sake of completeness.

In the case that $\mathcal{M}$ is $L^{\infty}[0, \alpha), 0<\alpha \leq \infty$, the proposition which follows may be found in [9], Proposition 1.2. By replacing the submajorisation inequality given in [28, Theorem II.3.1, by its non-commutative counterpart given in [12], Theorem 3.4 , the details of the proof are almost identical to those given in [9] and will be omitted.

Proposition 6.11. If $x \in \Lambda_{\phi}(\tau),\left\{y_{n}\right\}_{n=1}^{\infty} \in \Lambda_{\phi}(\tau)$ and the sequence $\left\{y_{n}\right\}_{n=1}^{\infty}$ converges to 0 for the measure topology, then

$$
\left\|x+y_{n}\right\|_{\Lambda_{\phi}(\tau)}-\|x\|_{\Lambda_{\phi}(\tau)}-\left\|y_{n}\right\|_{\Lambda_{\phi}(\tau)} \rightarrow_{n} 0 .
$$

Proposition 6.11 has the interesting consequence that, for sequences on the unit sphere of the Lorentz space $\Lambda_{\phi}(\tau)$, norm convergence coincides with measure convergence. This property is usually referred to as the Kadec-Klee property for convergence in measure.

Corollary 6.12. If $x \in \Lambda_{\phi}(\tau),\left\{x_{n}\right\}_{n=1}^{\infty} \in \Lambda_{\phi}(\tau)$, then the following statements are equivalent.

(i) $\left\|x-x_{n}\right\|_{\Lambda_{\phi}(\tau)} \rightarrow_{n} 0$.

(ii) $x_{n} \rightarrow x$ for the measure topology and $\left\|x_{n}\right\|_{\Lambda_{\phi}(\tau)} \rightarrow_{n}\|x\|_{\Lambda_{\phi}(\tau)}$.

Proof. Applying Proposition 6.11 with $y_{n}=x_{n}-x$, it follows that

$$
\left\|x_{n}\right\|_{\Lambda_{\phi}(\tau)}-\|x\|_{\Lambda_{\phi}(\tau)}-\left\|x_{n}-x\right\|_{\Lambda_{\phi}(\tau)} \rightarrow_{n} 0 .
$$

Since $\left\|x_{n}\right\|_{\Lambda_{\phi}(\tau)} \rightarrow_{n}\|x\|_{\Lambda_{\phi}(\tau)}$, it follows that $\left\|x_{n}-x\right\|_{\Lambda_{\phi}(\tau)} \rightarrow_{n} 0$. 
In the case that $\Lambda_{\phi}$ is separable, the preceding corollary follows from [9, Corollary 1.3, and [10], Theorem 2.9. The present approach is more direct.

The proposition which follows now shows that an arbitrary Lorentz space $\Lambda_{\phi}(\tau)$ has property $(W m)$.

Proposition 6.13. If $\left\{x_{n}\right\}_{n=1}^{\infty} \subseteq \Lambda_{\phi}(\tau), x \in \Lambda_{\phi}(\tau)$, then the following statements are equivalent.

(i) $x_{n} \rightarrow_{n} x$ in the weak topology $\sigma\left(\Lambda_{\phi}(\tau), \Lambda_{\phi}(\tau)^{\times}\right)$and in the measure topology.

(ii) $\left\|x_{n}-x\right\|_{\Lambda_{\phi}(\tau)} \rightarrow_{n} 0$.

Proof. It is only necessary to prove the implication (i) $\Longrightarrow$ (ii). Since the sequence $\left\{x_{n}\right\}_{n=1}^{\infty}$ is relatively $\sigma\left(\Lambda_{\phi}(\tau), \Lambda_{\phi}(\tau)^{\times}\right)$compact, it follows that the sequence $\left\{\mu\left(x_{n}\right)\right\}_{n=1}^{\infty} \subseteq \Lambda_{\phi}(m)$ is relatively $\sigma\left(\Lambda_{\phi}(m), \Lambda_{\phi}(m)^{\times}\right)$compact, as follows from [22], Theorem 5.2. By Smulian's theorem ([25], Chapter 5, Corollary 3.1.2), it may be assumed that there exists $z \in \Lambda_{\phi}(m)$ such that $\mu\left(x_{n}\right) \rightarrow_{n} z$ for the weak topology $\sigma\left(\Lambda_{\phi}(m), \Lambda_{\phi}(m)^{\times}\right)$. In particular,

$$
\int_{[s, t)} \mu\left(x_{n}\right) d m \rightarrow_{n} \int_{[s, t)} z d m
$$

for all $0<s<t$, since $\chi_{[s, t)} \in \Lambda_{\phi}(m)^{\times}$. Further, since $x_{n} \rightarrow_{n} x$ in the measure topology, it follows that $\mu\left(x_{n}\right) \rightarrow \mu(x)$ almost everywhere on $[0, \infty)$. Consequently, by the dominated convergence theorem, it follows that

$$
\int_{[s, t)} \mu\left(x_{n}\right) d m \rightarrow_{n} \int_{[s, t)} \mu(x) d m
$$

for all $0<s<t$. It follows directly that $\mu(x)=z$. Since $\phi^{\prime} \in \Lambda_{\phi}(m)^{\times}$, it follows immediately that

$$
\left\|x_{n}\right\|_{\Lambda_{\phi}(\tau)}=\int_{[0, \infty)} \mu\left(x_{n}\right) \phi^{\prime} d m \rightarrow_{n} \int_{[0, \infty)} \mu(x) \phi^{\prime} d m=\|x\|_{\Lambda_{\phi}(\tau)} .
$$

By Corollary 6.12, it now follows that $\left\|x_{n}-x\right\|_{\Lambda_{\phi}(\tau)} \rightarrow_{n} 0$.

For Lorentz spaces on the interval $[0, \alpha), 0<\alpha \leq \infty$, the preceding proposition may be found in [9], Corollary 1.3.

Since convergence for the weak topology $\sigma\left(\Lambda_{\phi}(\tau), \Lambda_{\phi}(\tau)^{*}\right)$ implies that for the weaker topology $\sigma\left(\Lambda_{\phi}(\tau), \Lambda_{\phi}(\tau)^{\times}\right)$, the following consequence is immediate.

Corollary 6.14. Each Lorentz space $\Lambda_{\phi}(\tau)$ has property $(W m)$.

Proposition 6.10 together with Corollary 6.14 now has the interesting consequence that if $\tau(\mathbf{1})<\infty$, then the relatively weakly compact subsets of the Lorentz space $\Lambda_{\phi}(\tau)$ are precisely those which are of uniformly absolutely continuous norm.

Corollary 6.15. If $\tau(\mathbf{1})<\infty$, then each weakly relatively compact subset of $\Lambda_{\phi}(\tau)$ is of uniformly absolutely continuous norm.

Note that by taking $\phi(t)=t, t \geq 0$, the preceding proposition is a proper extension of the Akemann characterisation of relatively weakly compact sets in $L_{1}(\tau)$ given by the equivalence $(\mathrm{i}) \Longleftrightarrow$ (iii) of Theorem 4.4 in the case that $\tau(\mathbf{1})<\infty$.

In the case of (commutative) Lorentz spaces on the interval $[0,1]$, the preceding proposition may be found in [8], Theorem 1. If the condition that $\tau(\mathbf{1})<\infty$ is 
omitted, then Corollary 6.15 may fail, even in the case that $\mathcal{M}$ is commutative, as the discussion which follows shows. Some preparations are necessary.

The observation which follows is due to D. Zanin (personal communication).

Proposition 6.16. Suppose that $E=E[0, \infty) \subseteq S(m)$ is a (strongly) symmetric space with order continuous norm. If each relatively weakly compact subset of $E$ is of uniformly absolutely continuous norm, then $E \subseteq L_{1}$.

Proof. Suppose that $E \nsubseteq L^{1}$. Let $x_{n}:=\chi_{[n, n+1)}, n \in \mathbb{N}$, and observe that $x_{n} \rightarrow_{n} 0$ for the $\sigma\left(E, E^{*}\right)=\sigma\left(E, E^{\times}\right)$topology. If this is not the case, then it may be assumed that there exist $\epsilon>0$, an element $0 \leq y \in E^{\times}$and a sequence $j(k) \uparrow_{k} \subseteq \mathbb{N}$ such that

$$
\int_{[0, \infty)} y x_{j(k)} d m=\int_{[j(k), j(k)+1)} y d m \geq \epsilon, \quad k \in \mathbb{N} .
$$

Since $\left|\int_{A} z d m\right| \leq \int_{[0, m(A))} \mu(z) d m$ for all $z \in S(m)$ and measurable subsets $A \subseteq$ $[0, \infty)$, it follows that, for each $n \in \mathbb{N}$,

$$
n \epsilon \leq \sum_{k=1}^{n} \int_{[j(k), j(k)+1)} y d m \leq \sum_{k=1}^{n} \int_{[0,1)} \mu\left(y \chi_{[j(k), j(k)+1)}\right) d m \leq \int_{[0, n)} \mu(y) d m,
$$

where the last inequality follows from [24, Lemma 16. As is easily seen,

$$
\lim _{t \rightarrow \infty} \mu(t ; y)=\lim _{n \rightarrow \infty} \frac{1}{n} \int_{[0, n)} \mu(y) d m
$$

and it follows immediately that

$$
\lim _{t \rightarrow \infty} \mu(t ; y) \geq \epsilon
$$

This implies that $\epsilon \mathbf{1} \leq \mu(y) \in E^{\times}$and consequently $L^{\infty} \subseteq E^{\times}$. It follows that $E \subseteq E^{\times \times} \subseteq L^{1}$, and this is a contradiction. In particular, the set $\left\{x_{n}\right\}_{n \in \mathbb{N}}$ is a relatively weakly compact subset of $E$. On the other hand, the sequence $\left\{x_{n}\right\}_{n=1}^{\infty}$ is not of uniformly absolutely continuous norm. Indeed, observe that

Consequently,

$$
x_{n} \chi_{[m, \infty)}= \begin{cases}0, & \text { if } n<m \\ x_{n}, & n \geq m\end{cases}
$$

$$
\sup _{n \in \mathbb{N}}\left\|x_{n} \chi_{[m, \infty)}\right\|_{E}=\sup _{n \geq m}\left\|x_{n}\right\|_{E}=\left\|x_{1}\right\|_{E},
$$

and this suffices to complete the proof of the proposition.

Lemma 6.17. The following statements are equivalent.

(i) $\Lambda_{\phi}(m) \nsubseteq L^{1}(m)$.

(ii) $\lim _{t \rightarrow \infty} \phi(t) / t=0$.

Proof. It only needs to be noted that

$$
\mathbf{1} \in \Lambda_{\phi}(m)^{\times}=M_{\psi}(m) \Longleftrightarrow \sup _{0<t<\infty} t / \phi(t)<\infty \Longleftrightarrow \inf _{0<t<\infty} \phi(t) / t>0 .
$$

Consequently,

$$
L^{\infty}(m) \subseteq M_{\psi}(m) \Longleftrightarrow \lim _{t \rightarrow \infty} \phi(t) / t=\inf _{0<t<\infty} \phi(t) / t>0,
$$

and so

$$
\Lambda_{\phi}(m)=M_{\psi}(m)^{\times} \not L^{1}(m) \Longleftrightarrow \lim _{t \rightarrow \infty} \phi(t) / t=0 .
$$


It follows from Proposition 6.16 and Lemma 6.17 that if $\lim _{t \rightarrow \infty} \phi(t) / t=0$, for example if $\phi(t)=t^{1 / 2}, t \geq 0$, then it is not the case that every relatively weakly compact subset in the Lorentz space $\Lambda_{\phi}(\tau)$ is of uniformly absolutely continuous norm. Further, since each Lorentz space on $[0, \infty)$ has property $(W m)$ (see Corollary 6.14), it follows that the implication (i) $\Longrightarrow$ (ii) of Proposition 6.10 fails in the case that $\tau(\mathbf{1})=\infty$, even in the case that $\mathcal{M}$ is commutative.

We mention a further consequence of Corollary 6.14.

Recall (see [28]) that the norm on $L^{1}+L^{\infty}(m)$ is given by the equality

$$
\|x\|_{L^{1}+L^{\infty}}=\int_{[0,1)} \mu(x) d m, \quad x \in L^{1}+L^{\infty}(m) .
$$

Consequently, the space $L^{1}+L^{\infty}(m)$ is just the Lorentz space $\Lambda_{\phi}(m)$ where

$$
\phi(t)= \begin{cases}t, & \text { if } 0 \leq t<1 \\ 1, & t \geq 1\end{cases}
$$

Recall that, in this case, the Köthe dual $\Lambda_{\phi}^{\times}(m)$ is given by $\Lambda_{\phi}^{\times}(m)=L^{1} \cap L^{\infty}(m)$.

Corollary 6.18. The space $L^{1}(\tau)+\mathcal{M}$ has property $(W m)$ for all semifinite $(\mathcal{M}, \tau)$.

Corollary 6.18 is noted in [30. Observe that $L^{1}+L^{\infty}$ is not separable, and so the norm on $L^{1}+L^{\infty}$ is not order continuous. In particular, $L^{1}+L^{\infty}$ is not a KB-space. This implies that Lemma 6.9 (i) fails if the condition that $\tau(\mathbf{1})<\infty$ is omitted.

Orlicz spaces on the interval $[0,1)$ with property $(W m)$ have been characterised in [3]. Let $\Phi$ be an increasing convex function on $[0, \infty)$ such that $\Phi(0)=0$, let $L_{\Phi}$ be the corresponding Orlicz space equipped with the norm

$$
\|x\|_{L_{\Phi}}=\inf \left\{\lambda: \lambda>0, \int_{[0,1)} \Phi(|x(t)| / \lambda) d m \leq 1\right\},
$$

and let $\Psi$ be the complementary function. It is shown in [3] that $L_{\Phi}$ has property $(W m)$ if and only if either $L_{\Phi}=L^{1}$ or the complementary function $\Psi$ satisfies $\lim _{t \rightarrow \infty} \Psi(C t) / \Psi(t)=\infty$ for some constant $C>0$. The class of Orlicz spaces which satisfies this latter condition is denoted by $\Delta_{3}$. If this characterisation is combined with the lifting theorem of [29], then this characterisation yields the following result.

Proposition 6.19. The Orlicz space $L_{\Phi}(\tau)$ has property $(W m)$ for all semifinite $(\mathcal{M}, \tau)$ with $\tau(\mathbf{1})<\infty$ if and only if $L_{\Phi}=L^{1}[0, \tau(\mathbf{1}))$ or $L_{\Phi} \in\left(\Delta_{3}\right)$.

Finally, it should be observed that there is a very large class of spaces which do not have property $(W m)$. The results which follow may be found in [29].

Proposition 6.20. Suppose that $\mathcal{M}$ is non-atomic. If $E, E^{\times} \subseteq S(\tau)$ have order continuous norms, then $E$ does not have property $(W m)$.

Proof. Since the norm on $E$ is order continuous, it follows that the Banach dual $E^{*}$ coincides with $E^{\times}$. Since $\mathcal{M}$ is non-atomic, there exists a sequence of projections $\left\{e_{n}\right\}_{n=1}^{\infty} \subseteq P(\mathcal{M})$ such that $e_{n} \downarrow_{n} 0$ and $\tau\left(e_{n}\right) \downarrow_{n} 0$. Set $x_{n}=e_{n} /\left\|e_{n}\right\|_{E}$. It is clear that $\left\|x_{n}\right\|_{E}=1, n \in \mathbb{N}$, and that $x_{n} \rightarrow_{n} 0$ for the measure topology. Now observe that $x_{n} \rightarrow_{n} 0$ for the weak topology. Indeed, if $0 \leq z \in E^{\times}$, then

$$
\left|\tau\left(x_{n} z\right)\right|=\left|\tau\left(x_{n} e_{n} z\right)\right| \leq\left\|e_{n} z\right\|_{E^{\times}} \rightarrow_{n} 0
$$


by Theorem 3.1 since the norm on $E^{\times}$is order continuous. If $E$ were to have property $(W m)$, then it would follow that $\left\|x_{n}\right\|_{E} \rightarrow_{n} 0$, but this is clearly a contradiction.

The following consequence now follows immediately from Proposition 6.20.

Corollary 6.21. If $\mathcal{M}$ is non-atomic and if $E \subseteq S(\tau)$ is reflexive, then $E$ does not have property $(W m)$.

A special case of the preceding corollary is that if $\mathcal{M}$ is non-atomic, and if $1<p<\infty$, then $L_{p}(\tau)$ does not have property $(W m)$. Although not covered by the preceding corollary, it is not difficult to show, by a similar argument, that the Schatten classes $\mathfrak{S}_{p}$ do not have property $(W m)$. Indeed, this follows from the following proposition.

Proposition 6.22. Suppose that $\mathcal{M}$ is atomic and all minimal projections have equal trace. Let $E \subseteq S(\tau)$ be strongly symmetric and suppose that $\sup \left\{\|e\|_{E}\right.$ : $e \in P(\mathcal{M})\}=\infty$. If $E, E^{\times}$have order continuous norms, then $E$ does not have property $(W m)$.

Proof. The stated assumptions imply that there exists a sequence of mutually orthogonal projections $\left\{e_{n}\right\}_{n=1}^{\infty} \subseteq P(\mathcal{M})$ such that $\left\|e_{n}\right\|_{E} \uparrow_{n} \infty$. Set $x_{n}:=$ $e_{n} /\left\|e_{n}\right\|_{E}$ for all $n \in \mathbb{N}$. Observe that $\left\|x_{n}\right\|_{E}=1, n \in \mathbb{N}$, and that $\mu\left(x_{n}\right)=$ $\chi_{\left[0, \tau\left(e_{n}\right)\right)} /\left\|e_{n}\right\|_{E} \rightarrow_{n} 0$, uniformly on $[0, \infty)$, so that $x_{n} \rightarrow_{n} 0$ for the measure topology. On the other hand, if $0 \leq z \in E^{\times}=E^{*}$, then

$$
\tau\left(x_{n} z\right)=\tau\left(z x_{n} \bigvee_{k \geq n} e_{k}\right) \leq\left\|z \bigvee_{k \geq n} e_{k}\right\|_{E^{\times}} \rightarrow_{n} 0
$$

by order continuity of the norm on $E^{\times}$and the fact that $\bigvee_{k \geq n} e_{k} \downarrow_{n} 0$. This shows that $E$ does not have property $(W m)$.

\section{LifTing OF PROPERTY $(W m)$}

It is shown in 30] that $E(\tau)$ has property $(W m)$ whenever $E \subset S(m)$ is a symmetric space with order continuous norm. Since [30] is not generally available, we will now outline a proof of this result for the sake of completeness and for the convenience of the reader. We need some technical preparation.

Lemma 7.1. Suppose that $E \subseteq S(m)$ is a symmetric space with order continuous norm. If $\left\{x_{n}\right\}_{n=1}^{\infty} \subseteq E(\tau)$ converges to 0 for the measure topology, then there exist a sequence $\left\{p_{n}\right\}_{n=1}^{\infty} \subseteq P(\mathcal{M})$ of mutually orthogonal projections and a subsequence $\left\{x_{k(n)}\right\}_{n=1}^{\infty} \subseteq\left\{x_{n}\right\}_{n=1}^{\infty}$ such that

$$
\left\|x_{k(n)}-x_{k(n)} p_{n}\right\|_{E(\tau)} \rightarrow_{n} 0 .
$$

If, in addition, $x_{n}^{*}=x_{n}, n \geq 1$, then

$$
\left\|x_{k(n)}-p_{n} x_{k(n)} p_{n}\right\|_{E(\tau)} \rightarrow_{n} 0 .
$$

Proof. The first assertion is proved in [10], Theorem 2.5. Now suppose in addition that $x_{n}^{*}=x_{n}, n \geq 1$, and that (17.1) holds. It follows that

$$
\left\|x_{k(n)}-p_{n} x_{k(n)}\right\|_{E(\tau)}=\left\|\left(x_{k(n)}-x_{k(n)} p_{n}\right)^{*}\right\|_{E(\tau)} \rightarrow_{n} 0
$$


so that

$$
\begin{aligned}
\left\|x_{k(n)}-p_{n} x_{k(n)} p_{n}\right\|_{E(\tau)} & \leq\left\|x_{k(n)}-x_{k(n)} p_{n}\right\|_{E(\tau)}+\left\|\left(p_{n} x_{k(n)}-x_{k(n)}\right) p_{n}\right\|_{E(\tau)} \\
& \leq 2\left\|x_{k(n)}-x_{k(n)} p_{n}\right\|_{E(\tau)} \rightarrow_{n} 0
\end{aligned}
$$

We shall need the following lemma, which follows from [7.

Lemma 7.2. Suppose that $\mathcal{M}$ is non-atomic and that $\tau(\mathbf{1})<\infty$. Suppose that $\left\{x_{n}\right\}_{n=1}^{\infty} \subseteq L^{1}(\tau)$ satisfies $x_{n}^{*}=x_{n}$ for all $n \in \mathbb{N}$ and that $x_{n} x_{m}=0, n \neq$ $m$. There exists a non-atomic commutative von Neumann subalgebra $\mathcal{R} \subseteq \mathcal{M}$ such that $\left\{x_{n}\right\}_{n=1}^{\infty} \subseteq L^{1}\left(\left.\tau\right|_{\mathcal{R}}\right)$, and there exists a normal trace-preserving unital *-isomorphism $\pi: L^{\infty}(0, \tau(\mathbf{1})) \rightarrow \mathcal{R}$.

In what follows, some facts concerning conditional expectations will be required. Let $\mathcal{N} \subseteq \mathcal{M}$ be a von Neumann subalgebra which is proper in the sense that the restriction $\tau_{\mathcal{N}}$ of the trace $\tau$ to $\mathcal{N}$ is semifinite. For each $x \in L^{1}(\tau)+\mathcal{M}$, there exists a uniquely determined element $\mathcal{E}_{\mathcal{N}}(x) \in L^{1}\left(\tau_{\mathcal{N}}\right)+\mathcal{N}$ called the conditional expectation with respect to $\mathcal{N}$ such that

$$
\tau(x y)=\tau\left(\mathcal{E}_{\mathcal{N}}(x) y\right), \quad \forall y \in L^{1}\left(\tau_{\mathcal{N}}\right) \cap \mathcal{N} .
$$

Suppose that $\mathcal{N} \subseteq \mathcal{M}$ is a proper von Neumann subalgebra and let $\mathcal{E}: L^{1}(\tau)+$ $\mathcal{M} \rightarrow L^{1}\left(\tau_{\mathcal{N}}\right)+\mathcal{N}$ be the conditional expectation. If $E \subseteq S(\tau)$ is a fully symmetric space, then $\mathcal{E}_{\mathcal{N}}: E \rightarrow E \cap S\left(\tau_{\mathcal{N}}\right)$ is a positive contractive projection. Further,

$$
\tau(\mathcal{E}(x) y)=\tau(x \mathcal{E}(y)), \quad x \in E, \quad y \in E^{\times} .
$$

The following theorem is established in 30] for general semifinite von Neumann algebras in the case that $E \subseteq S(m)$ has order continuous norm. To avoid technicalities, we consider here the case that $\tau(\mathbf{1})<\infty$, although the details in the more general case are essentially the same.

Theorem 7.3. Suppose that $E \subseteq S(0, \tau(\mathbf{1}))$ is strongly symmetric and that $\tau(\mathbf{1})<$ $\infty$. If $E$ has property $(W m)$, then $E(\tau)$ has property $(W m)$.

Proof. Since $E$ has property $(W m)$, and since $\tau(\mathbf{1})<\infty$, it follows from Lemma 6.9 that $E$ is a $K B$-space. In particular, the norm on $E$, and so also the norm on $E(\tau)$, is order continuous so that the Banach dual $E^{*}(\tau)$ coincides with the Köthe dual $E(\tau)^{\times}=E^{\times}(\tau)$. Assume first that $\mathcal{M}$ is non-atomic. Suppose then that $\left\{x_{n}\right\}_{n=1}^{\infty} \subseteq E(\tau)$ is such that $x_{n} \rightarrow_{n} 0$ for the measure topology and that $x_{n} \rightarrow_{n} 0$ for the weak topology $\sigma\left(E(\tau), E^{\times}(\tau)\right)$. By passing to real and imaginary parts, it is readily seen that it may be assumed that $x_{n}=x_{n}^{*}, n=1,2, \ldots$. If it is not the case that $\left\|x_{n}\right\|_{E(\tau)} \rightarrow_{n} 0$, then it may be assumed that there exists $\epsilon>0$ such that $\left\|x_{n}\right\|_{E(\tau)} \geq \epsilon$ for all $n \in \mathbb{N}$. Passing to a subsequence and relabelling if necessary, and using Lemma 7.1, it may be assumed that there exists a sequence $\left\{p_{n}\right\}_{n=1}^{\infty} \subseteq P(\mathcal{M})$ such that $\left\|x_{n}-p_{n} x_{n} p_{n}\right\|_{E(\tau)} \rightarrow_{n} 0$. Setting $y_{n}=p_{n} x_{n} p_{n}$ for all $n \in \mathbb{N}$, it is clear that the sequence $\left\{y_{n}\right\}_{n=1}^{\infty}$ converges to 0 for both the measure and weak topologies and that $\left\|y_{n}\right\|_{E(\tau)} \geq \epsilon$ for all sufficiently large $n \in \mathbb{N}$.

By Lemma 7.2, there exists a non-atomic commutative von Neumann subalgebra $\mathcal{R} \subseteq \mathcal{M}$ such that $\left\{x_{n}\right\}_{n=1}^{\infty} \subseteq L^{1}\left(\left.\tau\right|_{\mathcal{R}}\right)$, and there exists a normal trace preserving unital *-isomorphism $\pi: L^{\infty}(0, \tau(\mathbf{1})) \rightarrow \mathcal{R}$, which has a unique extension to a tracepreserving unital $*$-isomorphism, again denoted by $\pi$, from $S(0, \tau(\mathbf{1}))$ onto $S(\mathcal{R}, \tau)$, which preserves the singular value function. The mapping $\pi$ induces isometric 
*-isomorphisms, denoted again by $\pi$, from $E$, respectively $E^{\times}$, onto the closed subspaces $S(\mathcal{R}, \tau) \cap E(\tau)$, respectively $S(\mathcal{R}, \tau) \cap E(\tau)^{\times}$. Setting $f_{n}=\pi^{-1}\left(y_{n}\right) \in$ $E, n \in \mathbb{N}$, it follows immediately that $\mu\left(f_{n}\right)=\mu\left(y_{n}\right) \rightarrow_{n} 0$, and it is readily verified that $f_{n} \rightarrow_{n} 0$ for the weak topology on $E$. By assumption, $E$ has property $(W m)$ and so $\left\|f_{n}\right\|_{E} \rightarrow_{n}$ 0. However, since $\left\|y_{n}\right\|_{E(\tau)}=\left\|\mu\left(y_{n}\right)\right\|_{E}=\left\|f_{n}\right\|_{E}$, this contradicts the fact that $\left\|y_{n}\right\|_{E} \geq \epsilon$, for all sufficiently large $n \in \mathbb{N}$.

To reduce the general case to the non-atomic setting, let $\mathcal{N}$ be the von Neumann tensor product $\mathcal{M} \otimes L_{\infty}[0,1)$ equipped with the tensor product trace $\sigma=\tau \otimes m$, where $m$ denotes Lebesgue measure. Since $\mathcal{N}$ is non-atomic, it follows from the first part that $E(\sigma)$ has property $(W m)$. Suppose that $\left\{x_{n}\right\}_{n=1}^{\infty} \subset E(\tau)$ converges to 0 for both the measure and weak topologies. Observing that $\mu(x \otimes \mathbf{1})=\mu(x)$ for all $x \in S(\tau)$, it follows that $x_{n} \otimes \mathbf{1} \rightarrow_{n} 0$ holds for the measure topology in $S(\sigma)$. It will suffice therefore to show that $x_{n} \otimes \mathbf{1} \rightarrow_{n} 0$ for the weak topology on $E(\sigma)$, that is,

$$
\sigma\left(\left(x_{n} \otimes \mathbf{1}\right) y\right) \rightarrow_{n} 0, \quad y \in E(\sigma)^{*}=E(\sigma)^{\times} .
$$

Observing that the von Neumann subalgebra $\mathcal{M} \otimes \mathbf{1}$ is a proper von Neumann subalgebra of the von Neumann algebra $\mathcal{N}$, denote by $\mathcal{E}: \mathcal{N} \rightarrow \mathcal{M} \otimes \mathbf{1}$ the conditional expectation. Observe that if $y \in E(\sigma)^{\times}=E^{\times}(\sigma)$, then there exists $z \in E^{\times}(\tau)$ such that $\mathcal{E}(y)=z \otimes \mathbf{1}$. It follows that, for all $x \in E(\tau)$,

$$
\begin{aligned}
\sigma((x \otimes \mathbf{1}) y) & =\sigma(\mathcal{E}(x \otimes \mathbf{1}) y)=\sigma((x \otimes \mathbf{1}) \mathcal{E}(y)) \\
& =\sigma((x \otimes \mathbf{1})(y \otimes \mathbf{1}))=\tau(x y),
\end{aligned}
$$

and this suffices to complete the proof.

\section{Domination By COMPletely POSITIVE COMPACT OPERATORS}

We suppose that $(\mathcal{M}, \tau)$ is a semifinite von Neumann algebra acting in some Hilbert space $\mathcal{H}$. For each $n \in \mathbb{N}$, we let $\mathcal{M}_{n}=\mathcal{M} \bar{\otimes} M_{n}(\mathbb{C})$ be the von Neumann algebra tensor product acting in the tensor product Hilbert space $\mathcal{H} \otimes \mathbb{C}^{n}$ and with the tensor product trace $\tau_{n}:=\tau \otimes \operatorname{tr}_{n}$. Here $\operatorname{tr}_{n}$ denotes the standard matrix trace. As is well known (see, for example, [42, Chapter IV, Proposition 1.6), the von Neumann algebra tensor product $\mathcal{M}_{n}$ coincides with the algebraic tensor product $\mathcal{M} \otimes M_{n}(\mathbb{C})$ and may be identified with the space $M_{n}(\mathcal{M})$ of all $n \times n$-matrices $\left[x_{i j}\right]_{i, j=1}^{n}$ with values in $\mathcal{M}$.

Suppose now that $(\mathcal{N}, \sigma)$ is a semifinite von Neumann algebra, that $E \subseteq S(\tau)$, $F \subseteq S(\sigma)$ are strongly symmetric spaces and that $T: E \rightarrow F$ is a linear mapping. For each $n \in \mathbb{N}$, the space of all $n \times n$-matrices with entries in $E$ (respectively $F$ ) will be denoted by $M_{n}(E)$ (respectively $M_{n}(F)$ ).

For each $n \in \mathbb{N}$, we let $T_{n}: M_{n}(E) \rightarrow M_{n}(F)$ be defined by setting

$$
T_{n}\left(\left[x_{i j}\right]_{i, j=1}^{n}\right)=\left[T\left(x_{i j}\right)\right]_{i, j=1}^{n},
$$

for all $\left[x_{i j}\right]_{i, j=1}^{n} \in M_{n}(E)$. The mapping $T$ is said to be completely positive if and only if $T_{n} \geq 0$ for every $n \in \mathbb{N}$; that is, $T_{n}$ maps $M_{n}(E) \cap S\left(\tau_{n}\right)_{+}$into $M_{n}(F) \cap$ $S\left(\sigma_{n}\right)_{+}$, for each $n \in \mathbb{N}$. Denote by $C P(E, F)$ the collection of all completely positive maps $T: E \rightarrow F$. If $T \in C P(E, F)$, then we will write $0 \leq_{\text {cp }} T: E \rightarrow F$. If $S, T \in C P(E, F)$, then we write $0 \leq_{\mathrm{cp}} S \leq_{\mathrm{cp}} T$ if and only if $0 \leq_{\mathrm{cp}} T-S$. Note that if $E, F \subseteq S(m)$ are strongly symmetric spaces on $[0, \infty)$, then via the identifications $E\left(\tau_{n}\right)=M_{n}(E(\tau)), F\left(\sigma_{n}\right)=M_{n}(F(\sigma))$, each of the mappings $T_{n}$ defined above induces a linear mapping from $E\left(\tau_{n}\right)$ to $F\left(\sigma_{n}\right)$. Without risk of 
confusion, we continue to denote these mappings by $T_{n}$. In this setting, the linear mapping $T: E(\tau) \rightarrow F(\sigma)$ is completely positive if and only if each of the mappings $T_{n}: E\left(\tau_{n}\right) \rightarrow F\left(\sigma_{n}\right)$ is positive in the usual sense, that is, $0 \leq T_{n}(x) \in F\left(\sigma_{n}\right)$ whenever $0 \leq x \in E\left(\tau_{n}\right)$. Further details may be found in 18 .

It should be observed that if $E \subseteq S(\tau)$ is a strongly symmetric space, and if $\varphi \in E^{*}$, then $\varphi \in E^{\times}$if and only if, whenever $x_{\alpha} \downarrow_{\alpha} 0$ in $E$, it follows that $\varphi\left(x_{\alpha}\right) \rightarrow_{\alpha} 0$ (see e.g. [15], Theorem 5.11).

Recall (see [18) that if $E \subseteq S(\tau), F \subseteq S(\sigma)$ are strongly symmetric spaces, then the continuous linear mapping $T: E \rightarrow F$ will be called order continuous if $\sigma\left(T\left(x_{\alpha}\right) z\right) \rightarrow_{\alpha} 0$ whenever $x_{\alpha} \downarrow_{\alpha} 0$ in $E$ and $0 \leq z \in F^{\times}$.

Using the above remark, it follows that if $T: E \rightarrow F$ is order continuous, and if $T^{*}: F^{*} \rightarrow E^{*}$ denotes the Banach adjoint mapping, then $T^{*} z \in E^{\times}$whenever $z \in F^{\times}$. In this case, the restriction of the adjoint $T^{*}$ to the Köthe dual $F^{\times}$will be denoted by $T^{\times}$so that $T^{\times}: F^{\times} \rightarrow E^{\times}$. It is clear that if $T \geq 0$, then $T^{\times} \geq 0$. If $0 \leq T: E \rightarrow F$, then $T$ is order continuous if and only if $x_{\alpha} \downarrow_{\alpha} 0$ in $E$ implies $T x_{\alpha} \downarrow_{\alpha} 0$ in $F$. It follows, in particular, that if $0 \leq S, T: E \rightarrow F$ are positive linear mappings with $0 \leq S \leq T$, then $S$ is order continuous if $T$ is order continuous.

The following results are proved in [18, Theorem 5.5 and Corollary 5.6, and are stated for the convenience of the reader.

Theorem 8.1. Let $E, F \subseteq S(m)$ be strongly symmetric. Let $0 \leq S, T: E(\tau) \rightarrow$ $F(\sigma)$ be linear mappings and suppose that $0 \leq_{\mathrm{cp}} S \leq_{\mathrm{cp}} T$. If $T$ is order continuous, if the norms on $E^{\times}$and $F$ are order continuous, and if $T$ is compact, then $S$ is compact.

Corollary 8.2. Let $E \subseteq S(m)$ be strongly symmetric and suppose that $0 \leq S, T$ : $E(\tau) \rightarrow E(\tau)$ are linear mappings which satisfy $0 \leq_{c p} S \leq_{c p} T$. If the norms on $E, E^{\times}$are order continuous and if $T$ is compact, then $S$ is compact.

We begin with the following complement to [18, Theorem 5.5. Here $(\mathcal{M}, \tau)$, $(\mathcal{N}, \sigma),(\mathcal{R}, \eta)$ denote semifinite von Neumann algebras.

Theorem 8.3. Suppose that $E \subseteq S(\tau), F \subseteq S(\sigma), G \subseteq S(\eta)$ are strongly symmetric and that $0 \leq S_{1}, T_{1}: E \rightarrow F, 0 \leq S_{2}, T_{2}: F \rightarrow G$ are linear mappings which satisfy $0 \leq_{c p} S_{i} \leq_{c p} T_{i}, i=1,2$. If the norms on $F, G$ are order continuous, and if $T_{i}, i=1,2$, is compact, then $S_{2} \circ S_{1}$ is compact.

Proof. Since $T_{1}$ is compact and the norm on $F$ is order continuous, it follows from Proposition 5.1 that (denoting $B(E)$ the unit ball of $E) T_{1}\left(B(E)_{ \pm}\right) \subseteq F$ is of uniformly absolutely continuous norm. Since $T_{1} \geq 0$, it follows that $T_{1}\left(B(E)_{ \pm}\right) \subseteq F_{+}$, and so $T_{1}(B(E))$ is of bi-uniformly absolutely continuous norm, by Proposition 3.15 . Since $0 \leq S_{1} \leq T_{1}$, it follows also that $S_{1}(B(E)) \subseteq F$ is also of bi-uniformly absolutely continuous norm. Let $\epsilon>0$ be given. By Theorem 3.23 and the remark following, there exist a positive constant $K$ and $e \in P(\mathcal{N}) \cap \mathcal{F}(\sigma)$ such that

$$
S_{1}(B(E)) \subseteq K e B(\mathcal{N}) e+\epsilon B(F) \subseteq K B(e \mathcal{N} e)+\epsilon B(F) .
$$

Since $e \in \mathcal{F}(\sigma) \subseteq F$, it follows that $e \mathcal{N} e \subseteq F$ and that $B(e \mathcal{N} e)$ is a bounded subset of $F$. If $j: e \mathcal{N} e \rightarrow F$ is the natural inclusion, it is clear that

$$
0 \leq_{c p} S_{2} \circ j \leq_{c p} T_{2} \circ j: e \mathcal{N} e \rightarrow G
$$


and that $T_{2} \circ j$ is compact. Since $G$ has order continuous norm, it follows from [18], Proposition 5.4, that the $S_{2} \circ j: e \mathcal{N} e \rightarrow G$ is compact. Observing that

$$
S_{2} \circ S_{1}(B(E)) \subseteq K S_{2} \circ j(B(e \mathcal{M} e))+\epsilon S_{2}(B(F)),
$$

it then follows that $S_{1} \circ S_{2}(B(E))$ is totally bounded in $G$, and hence $S_{1} \circ S_{2}$ is compact.

Corollary 8.4. Suppose that $E \subseteq S(\tau)$ is strongly symmetric and that $0 \leq S, T$ : $E \rightarrow E$ are linear mappings which satisfy $0 \leq_{c p} S \leq_{c p} T$. If the norm on $E$ is order continuous, and if $T$ is compact, then $S^{2}$ is compact.

Note that the preceding corollary is valid in the case that $E=L^{1}(\tau)$.

In what follows, it will be assumed that $E, F, G \subseteq S(m)$ are strongly symmetric spaces. Recall that if the norm on $E$ is order continuous, then the norm on $E(\tau)$ is order continuous and the Banach dual $E(\tau)^{*}$ coincides with the Köthe dual $E(\tau)^{\times}=E^{\times}(\tau)$. Note that if $F$ has order continuous norm and if $T: E(\tau) \rightarrow F(\sigma)$ is order continuous, then the Banach adjoint $T^{*}$ coincides with the mapping $T^{\times}$.

A dual version of Theorem 8.3 now follows. Together with Theorem 8.3 , this is a non-commutative analogue of [1], Theorem 16.15.

Theorem 8.5. Suppose that $E, F, G \subseteq S(m)$ are strongly symmetric and that $0 \leq S_{1}, T_{1}: E(\tau) \rightarrow F(\sigma), 0 \leq S_{2}, T_{2}: F(\sigma) \rightarrow G(\eta)$ are linear mappings such that $T_{i}, i=1,2$, is order continuous and which satisfy $0 \leq_{c p} S_{i} \leq_{c p} T_{i}, i=1,2$. If the norms on $E^{\times}, F^{\times}, G^{\times}$are order continuous, if $E, G$ have the Fatou property and if $T_{i}, i=1,2$, is compact, then $S_{2} \circ S_{1}$ is compact.

Proof. Observe first that order continuity of $T_{i}$ implies that of $S_{i}, i=1,2$. This in turn implies that $T_{1}^{\times}, S_{1}^{\times}: F(\sigma)^{\times} \rightarrow E(\tau)^{\times}$and that $T_{2}^{\times}, S_{2}^{\times}: G(\eta)^{\times} \rightarrow F(\sigma)^{\times}$. From [18, Proposition 3.7 (iii), it follows that $0 \leq_{\mathrm{cp}} S_{i}^{\times} \leq_{\mathrm{cp}} T_{i}^{\times}, i=1,2$. By Schauder's theorem, $T_{1}^{*}, T_{2}^{*}$, and hence $T_{1}^{\times}, T_{2}^{\times}$, are compact. Since the norms on $F(\sigma)^{\times}=F^{\times}(\sigma), E(\tau)^{\times}=E^{\times}(\tau)$ are order continuous, it follows from Theorem 8.3 that $S_{1}^{\times} \circ S_{2}^{\times}: G(\eta)^{\times} \rightarrow E(\tau)^{\times}$is compact. Observing that $S_{1}^{\times} \circ S_{2}^{\times}=$ $\left(S_{2} \circ S_{1}\right)^{\times}$, it follows from Schauder's theorem that $\left(S_{2} \circ S_{1}\right)^{\times *}: E(\tau)^{\times *} \rightarrow$ $G(\eta)^{\times *}$ is compact. Order continuity of the norms on $E(\tau)^{\times}, G(\eta)^{\times}$implies that $E(\tau)^{\times *}=E(\tau)^{\times \times}, G(\eta)^{\times *}=G(\eta)^{\times \times}$, and order continuity of the norm on $E^{\times}$implies that $\left(S_{2} \circ S_{1}\right)^{\times *}=\left(S_{2} \circ S_{1}\right)^{\times \times}$. Consequently, $\left(S_{2} \circ S_{1}\right)^{\times \times}$: $E(\tau)^{\times \times} \rightarrow G(\eta)^{\times \times}$is compact. Since $E, G$ have the Fatou property, it follows that $E(\tau)^{\times \times}=E(\tau), G(\eta)^{\times \times}=G(\eta)$, and it now follows that $S_{2} \circ S_{1}$ is compact.

Corollary 8.6. Suppose that $E \subseteq S(m)$ is strongly symmetric and that $0 \leq S, T$ : $E(\tau) \rightarrow E(\tau)$ are linear mappings such that $T$ is order continuous and which satisfy $0 \leq_{\mathrm{cp}} S \leq_{\mathrm{cp}} T$. If $E$ has the Fatou property, if the norm on $E^{\times}$is order continuous and if $T$ is compact, then $S^{2}$ is compact. $L^{\infty}$.

Observe that the assertion of the preceding theorem holds in the case that $E=$

\section{Domination by Completely positive Dunford-Pettis operators}

The bounded linear operator $T: E \rightarrow F$, with $E, F$ Banach spaces, is said to be a Dunford-Pettis operator if and only if $T$ maps relatively weakly compact subsets in $E$ into relatively compact sets in $F$. 
Theorem 9.1. Suppose that $\tau(\mathbf{1})<\infty$ and let $E, F \subseteq S(m)$ be strongly symmetric spaces such that $E$ has property $(W m)$ and $F$ has order continuous norm. If $0 \leq$ $S, T: E(\tau) \rightarrow F(\sigma)$ are linear mappings which satisfy $0 \leq_{c p} S \leq_{c p} T$ and if $T$ is a Dunford-Pettis operator, then $S$ is a Dunford-Pettis operator.

Proof. We suppose that $\mathcal{K} \subseteq E(\tau)$ is relatively weakly compact. Since $E$ has property $(W m)$, it follows from Lemma 6.9 (i) that the norm on $E$ is order continuous, and from Theorem 7.3 it follows that $E(\tau)$ has property $(W m)$. Using the assumption that $\tau(\mathbf{1})<\infty$, it now follows from Proposition 6.10 that $\mathcal{K}$ is of uniformly absolutely continuous norm in $E$. Let $\epsilon>0$ be given. By Theorem 3.12 , there exists $0 \leq h_{\epsilon} \in \mathcal{F}(\tau)$ such that

$$
\mathcal{K} \subseteq h_{\epsilon} B(\mathcal{M})+B(\mathcal{M}) h_{\epsilon}+\epsilon B(E(\tau)) \subseteq 2\left\|h_{\epsilon}\right\|_{\infty} B(\mathcal{M})+\epsilon B(E(\tau)) .
$$

Using the assumption that $\tau(\mathbf{1})<\infty$, let $j: \mathcal{M} \rightarrow E(\tau)$ be the natural embedding and note that

$$
0 \leq_{c p} S \circ j \leq_{c p} T \circ j: \mathcal{M} \rightarrow F(\sigma) .
$$

It follows from Proposition 4.3(iii) that $j(B(\mathcal{M})) \subseteq(-j \mathbf{1}, j \mathbf{1}) \subseteq E(\tau)$ is relatively weakly compact in $E(\tau)$. Since $T$ is a Dunford-Pettis operator, this implies that the map $T \circ j: \mathcal{M} \rightarrow F(\sigma)$ is compact. By [18, Proposition 5.4, it follows that the map $S \circ j: \mathcal{M} \rightarrow F(\sigma)$ is also compact. Since $S \circ j(B(\mathcal{M}))=S(B(\mathcal{M}))$, it follows that $S(B(\mathcal{M}))$ is relatively compact in $F(\sigma)$. We obtain, therefore, that

$$
S(\mathcal{K}) \subseteq 2\left\|h_{\epsilon}\right\|_{\infty} S(B(\mathcal{M}))+\epsilon S(B(E(\tau))),
$$

and this implies that $S(\mathcal{K}) \subseteq F(\sigma)$ is totally bounded and hence relatively compact. This suffices to establish the theorem.

Corollary 9.2. Suppose that $\tau(\mathbf{1})<\infty$. If $0 \leq S, T: L^{1}(\tau) \rightarrow L^{1}(\sigma)$ are linear mappings which satisfy $0 \leq_{c p} S \leq_{c p} T$ and if $T$ is a Dunford-Pettis operator, then $S$ is a Dunford-Pettis operator.

In the commutative setting, Theorem 9.1 reduces to a special case of a theorem of Kalton and Saab (see e.g. 1], Theorem 19.11 of Chapter 5). Corollary 9.2 was first proved in [17] in the case of abstract $L$-spaces. It seems to be an interesting question as to whether Theorem 9.1 continues to hold without the restriction that $\tau(\mathbf{1})<\infty$.

\section{BAND-LIKE DECOMPOSITIONS}

It is shown in 33, Corollary 3.7, that each completely positive mapping $T$ : $\mathcal{M} \rightarrow L^{p}(\sigma), 1 \leq p<\infty$, admits a unique decomposition $T=T_{1}+T_{2}$, with $0 \leq_{\mathrm{cp}} T_{i} \leq_{\mathrm{cp}} T, i=1,2$, with $T_{1}$ compact and such that $T_{2}$ dominates no non-zero compact operator (in the sense of complete positivity). Such a band decomposition in the setting of Banach lattices goes back to [17.

Before proceeding, it will be convenient to make some remarks concerning the order structure of the space $\mathcal{L}(E, F)$ of continuous linear operators from $E$ to $F$. Here, $E \subseteq S(\tau), F \subseteq S(\sigma)$ are strongly symmetric spaces. The space $\mathcal{L}(E, F)$ is an ordered vector space with the ordering induced by the natural cone $\mathcal{L}(E, F)_{+}$of positive operators $0 \leq T: E \rightarrow F$. Here $0 \leq T: E \rightarrow F$ if and only if $T x \geq 0$ for all $0 \leq x \in E$ or, equivalently, $\sigma(y T(x)) \geq 0$ for all $0 \leq x \in E, 0 \leq y \in F^{\times}$. Observe that the cone $\mathcal{L}(E, F)_{+}$is order complete in the sense of the following proposition. 
Proposition 10.1. If $0 \leq T_{\alpha} \uparrow S \subseteq \mathcal{L}(E, F)_{+}$, then $T=\sup _{\alpha} T_{\alpha}$ exists in $\mathcal{L}(E, F)_{+}$and

$$
T(x)=\sup _{\alpha} T_{\alpha}(x), \quad 0 \leq x \in E .
$$

Proof. For all $0 \leq x \in E$ observe that $T(x)=\sup _{\alpha} T_{\alpha}(x)$ exists in $F$ since $0 \leq$ $T_{\alpha}(x) \uparrow_{\alpha} \leq S(x)$. It is clear that $T(\lambda x)=\lambda T(x)$ for all scalars $0 \leq \lambda$ and that

$$
T\left(x_{1}+x_{2}\right) \leq T\left(x_{1}\right)+T\left(x_{2}\right)
$$

for all $x_{1}, x_{2} \in E$. To obtain the reverse inequality, suppose that $0 \leq y \in F^{\times}$, and let $\epsilon>0$ be given. There exist indices $\alpha_{1}, \alpha_{2}$ such that

$$
\sigma\left(y T_{\alpha_{1}}\left(x_{1}\right)\right) \geq \sigma\left(y T\left(x_{1}\right)\right)-\epsilon, \quad \sigma\left(y T_{\alpha_{2}}\left(x_{2}\right)\right) \geq \sigma\left(y T\left(x_{2}\right)\right)-\epsilon .
$$

Since $0 \leq T_{\alpha} \uparrow_{\alpha}$, there exists an index $\alpha_{3}$ such that $0 \leq T_{\alpha_{1}}, T_{\alpha_{2}} \leq T_{\alpha_{3}}$. Consequently,

$$
\begin{aligned}
\sigma\left(y T\left(x_{1}+x_{2}\right)\right) \geq \sigma\left(y T_{\alpha_{3}}\left(x_{1}+x_{2}\right)\right) & \geq \sigma\left(y T_{\alpha_{1}}\left(x_{1}\right)\right)+\sigma\left(y T_{\alpha_{2}}\left(x_{2}\right)\right) \\
& \geq \sigma\left(y T\left(x_{1}\right)\right)+\sigma\left(y T\left(x_{2}\right)\right)-2 \epsilon,
\end{aligned}
$$

and the assertion follows since $F_{+}^{\times}$separates the points of $F_{+}$.

Corollary 10.2. Suppose that $E \subseteq S(m), F \subseteq S(m)$ are strongly symmetric. If $0 \leq_{\mathrm{cp}} T_{\alpha} \uparrow_{\alpha} \leq_{\mathrm{cp}} S \in C P(E(\tau), F(\sigma))$, then $T=\sup _{\alpha} T_{\alpha} \in C P(E(\tau), F(\sigma))$ and $0 \leq\left(T_{\alpha}\right)_{n} \uparrow_{\alpha} T_{n} \leq S_{n} \in \mathcal{L}\left(E\left(\tau_{n}\right), F\left(\sigma_{n}\right)\right)$ for every $n \in \mathbb{N}$.

Proof. By Proposition 10.1 $T=\sup _{\alpha} T_{\alpha}$ exists in $\mathcal{L}(E(\tau), F(\sigma))_{+}$and $\sigma\left(y T_{\alpha}(x)\right) \rightarrow_{\alpha}$ $\sigma(y T(x))$ for every $x \in E(\tau), y \in F(\sigma)$. If $n \in \mathbb{N}$, then for every $0 \leq x=\left[x_{i j}\right]_{i, j=1}^{n} \in$ $E\left(\tau_{n}\right), 0 \leq y=\left[y_{i j}\right]_{i, j=1}^{n} \in F\left(\sigma_{n}\right)$,

$$
0 \leq \sigma_{n}\left(y\left(T_{\alpha}\right)_{n}(x)\right)=\sum_{i, k=1}^{n} \sigma\left(y_{i k} T_{\alpha}\left(x_{k i}\right)\right) \rightarrow_{\alpha} \sum_{i, k=1}^{n} \sigma\left(y_{i k} T\left(x_{k i}\right)\right)=\sigma_{n}\left(y T_{n}(x)\right) .
$$

Further, since

$$
0 \leq \sigma_{n}\left(y\left(T_{\alpha}\right)_{n}(x)\right) \leq \sigma_{n}\left(y S_{n}(x)\right)
$$

for every index $\alpha$, it follows also that

$$
0 \leq \sigma_{n}\left(y T_{n}(x)\right) \leq \sigma_{n}\left(y S_{n}(x)\right), \quad 0 \leq x \in E\left(\tau_{n}\right), 0 \leq y \in F\left(\sigma_{n}\right) .
$$

In particular, it follows that $0 \leq T_{n} \leq S_{n}$ and so $T$ is completely positive. The final assertion of the corollary now follows immediately from Proposition 10.1 and the fact that $\left(T_{\alpha}\right)_{n} \uparrow_{\alpha}$ for each $n \in \mathbb{N}$.

Proposition 10.3. Suppose that the norm on $F \subseteq S(\tau)$ is order continuous. If $0 \leq T_{\alpha} \uparrow_{\alpha} T \in \mathcal{L}(\mathcal{M}, F)$, and if $T_{\alpha}$ is compact for every index $\alpha$, then also $T$ is compact.

Proof. Since $T_{\alpha}(\mathbf{1}) \uparrow_{\alpha} T(\mathbf{1})$, and since the norm on $F$ is order continuous, it follows that there exists an index $\alpha_{0}$ such that

$$
\left\|T_{\alpha_{0}}(\mathbf{1})-T(\mathbf{1})\right\|_{F}<\epsilon / 2 .
$$

Since $T_{\alpha_{0}}$ is compact, there exists a finite subset $0 \leq x_{1}, x_{2}, \ldots, x_{n}$ such that

$$
T_{\alpha_{0}}\left(B(\mathcal{M})_{+}\right) \subseteq \bigcup_{i=1}^{n}\left(T_{\alpha_{0}}\left(x_{i}\right)+(\epsilon / 2) B(F)\right) .
$$


Now suppose that $0 \leq y \in B(\mathcal{M})_{+}$. Then there exists an index $i$ such that $\left\|T_{\alpha_{0}}\left(y-x_{i}\right)\right\|_{F}<\epsilon / 2$ and it follows that

$$
\begin{aligned}
\left\|T(y)-T_{\alpha_{0}}\left(x_{i}\right)\right\|_{F} & \leq\left\|T(y)-T_{\alpha_{0}}(y)\right\|_{F}+\left\|T_{\alpha_{0}}(y)-T_{\alpha_{0}}\left(x_{i}\right)\right\|_{F} \\
& \leq\left\|\left(T-T_{\alpha_{0}}\right)(y)\right\|_{F}+\epsilon / 2 \\
& \leq\left\|\left(T-T_{\alpha_{0}}\right)(\mathbf{1})\right\|_{F}+\epsilon / 2 \leq \epsilon .
\end{aligned}
$$

It follows that the image under $T$ of the positive part of the unit ball in $\mathcal{M}$ is totally bounded in $F$, and the compactness of $T$ now follows.

Proposition 10.4. Assume that $F \subseteq S(m)$ has order continuous norm. If $0 \leq$ $T: \mathcal{M} \rightarrow F(\sigma)$ is completely positive, then there exists a unique decomposition $T=T_{1}+T_{2}$ with $T_{1}, T_{2}: \mathcal{M} \rightarrow F(\sigma)$ completely positive and such that $T_{1}$ is compact and $T_{2}$ dominates no non-zero compact map (in the sense of complete positivity).

Proof. Set

$$
\mathcal{C}_{T}=\left\{S \in C P(\mathcal{M}, F(\sigma)): 0 \leq_{\mathrm{cp}} S \leq_{\mathrm{cp}} T, S \text { compact }\right\} .
$$

By Corollary 10.2 and Proposition 10.3, each chain in $\mathcal{C}_{T}$ has an upper bound. By Zorn's lemma, $\mathcal{C}_{T}$ has a maximal element $T_{1}$. The element $T_{1}$ is completely positive and compact and satisfies $0 \leq_{\mathrm{cp}} T_{1} \leq_{\mathrm{cp}} T$. The required decomposition is then obtained by setting $T_{2}=T-T_{1}$. That $T_{2}$ dominates no non-zero completely positive compact operator now follows from the maximality of $T_{1}$. To establish the uniqueness of the decomposition, suppose that $T=T_{1}^{\prime}+T_{2}^{\prime}$, with $T_{1}^{\prime}, T_{2}^{\prime}$ completely positive, $T_{1}^{\prime}$ compact, and for which $T_{2}^{\prime}$ dominates no non-zero compact map. By the maximality of $T_{1}$, it follows that $0 \leq_{\mathrm{cp}} T_{1}^{\prime} \leq_{\mathrm{cp}} T_{1}$. Consequently,

$$
0 \leq_{\mathrm{cp}} T_{1}-T_{1}^{\prime}=T_{2}^{\prime}-T_{2} \leq_{\mathrm{cp}} T_{2}^{\prime} .
$$

Since $0 \leq_{\mathrm{cp}} T_{1}-T_{2}$ is compact and $T_{2}^{\prime}$ dominates no non-zero completely positive compact operator, it now follows that $T_{1}=T_{1}^{\prime}$ and so also $T_{2}=T_{2}^{\prime}$.

The preceding proposition is contained in 33], Theorem 3.6, with $F=L^{q}(m)$, $1 \leq q<\infty$, and the proof given above is somewhat more direct, even in this special case. It is the non-commutative counterpart of [17, Theorem 3.4, in the setting of Banach lattices.

It might be remarked that if $0 \leq T: \mathcal{M} \rightarrow F \subseteq S(\sigma)$, and if one sets

$$
\mathcal{C}_{T}^{\prime}=\{0 \leq S \in \mathcal{L}(\mathcal{M}, F): 0 \leq S \leq T, S \text { compact }\},
$$

then Proposition 10.1 and the proof of Proposition 10.4 with $\mathcal{C}_{T}$ replaced by $\mathcal{C}_{T}^{\prime}$ yield the following.

Proposition 10.5. Assume that $F \subseteq S(\sigma)$ is strongly symmetric and has order continuous norm. If $0 \leq T \in \mathcal{L}(\mathcal{M}, F(\sigma))$, then there exists a unique decomposition $T=T_{1}+T_{2}$ with $0 \leq T_{1}, T_{2} \in \mathcal{L}(\mathcal{M}, F(\sigma))$, such that $T_{1}$ is compact and $T_{2}$ dominates no non-zero compact map.

Proposition 10.6. Let $E \subseteq S(\tau), F \subseteq S(\sigma)$ be strongly symmetric spaces. Suppose that $\tau(\mathbf{1})<\infty$, that $E$ has property $(W m)$ and that the norm on $F$ is order continuous. If $0 \leq T_{\alpha} \uparrow_{\alpha} T \in \mathcal{L}(E, F)$, and if $T_{\alpha}$ is a Dunford-Pettis operator for every index $\alpha$, then also $T$ is Dunford-Pettis. 
Proof. Suppose $\mathcal{K} \subseteq E$ is weakly compact and let $\epsilon>0$ be given. Since $E$ has property $(W m)$, it may be assumed, using Proposition 6.10, that $\mathcal{K} \subseteq C_{\epsilon} B(\mathcal{M})+$ $\epsilon B(E)$ for some constant $C_{\epsilon}>0$. Since the norm on $E$ is order continuous, it follows that $B(\mathcal{M})$ is weakly compact in $E$. Consequently, the restriction $\left.T_{\alpha}\right|_{\mathcal{M}}: \mathcal{M} \rightarrow F$ is compact for every index $\alpha$. Since $0 \leq 0 \leq\left.\left. T_{\alpha}\right|_{\mathcal{M}} \uparrow_{\alpha} T\right|_{\mathcal{M}} \subseteq \mathcal{L}(\mathcal{M}, F)$, it follows from Proposition 10.3 that $\left.T\right|_{\mathcal{M}}$ is compact. Since $\sup _{\alpha}\left\|T_{\alpha}\right\| \leq\|T\|$, it then follows that the image of $\mathcal{K}$ under $T$ is totally bounded in $F$, and this suffices to complete the proof of the proposition.

Using the fact that property $(W m)$ lifts from the commutative setting to the non-commutative setting by Theorem 7.3 , the same arguments as in the proofs of Propositions 10.4, 10.5 now yield the following consequences.

Proposition 10.7. Assume that $\tau(\mathbf{1})<\infty$, that $E \subseteq S(m)$ has property $(W m)$ and that $F \subseteq S(m)$ has order continuous norm. If $0 \leq T: E(\tau) \rightarrow F(\sigma)$ is completely positive, then there exists a unique decomposition $T=T_{1}+T_{2}$ with $T_{1}, T_{2}: E(\tau) \rightarrow F(\sigma)$ completely positive and such that $T_{1}$ is Dunford-Pettis and $T_{2}$ dominates no non-zero Dunford-Pettis operator (in the sense of complete positivity).

Proposition 10.8. Assume that $\tau(\mathbf{1})<\infty$, that $E \subseteq S(m)$ has property $(W m)$ and that $F \subseteq S(m)$ has order continuous norm. If $0 \leq T: E(\tau) \rightarrow F(\sigma)$, then there exists a unique decomposition $T=T_{1}+T_{2}$ with $0 \leq \bar{T}_{1}, T_{2}: E(\tau) \rightarrow F(\tau)$ such that $T_{1}$ is Dunford-Pettis and $T_{2}$ dominates no non-zero Dunford-Pettis operator.

Corollary 10.9. If $\tau(\mathbf{1})<\infty$, and if $0 \leq T: L^{1}(\tau) \rightarrow L^{1}(\sigma)$ (respectively, is completely positive), then there exists a unique decomposition $T=T_{1}+T_{2}$ with $0 \leq T_{1}, T_{2}: L^{1}(\tau) \rightarrow L^{1}(\sigma)$ (respectively, completely positive) such that $T_{1}$ is Dunford-Pettis and $T_{2}$ dominates no non-zero Dunford-Pettis operator (respectively, in the sense of complete positivity).

\section{ACKNOWLEDGEMENT}

Some of the results of this paper were announced by the first-named author at the conference Positivity VI, held in Escorial, Spain, in July 2009.

The authors wish to thank the referee for some useful comments.

\section{REFERENCES}

[1] Charalambos D. Aliprantis and Owen Burkinshaw, Positive operators, Pure and Applied Mathematics, vol. 119, Academic Press, Inc., Orlando, FL, 1985. MR809372 (87h:47086)

[2] Charles A. Akemann, The dual space of an operator algebra, Trans. Amer. Math. Soc. 126 (1967), 286-302. MR0206732 (34 \#6549)

[3] Sergey V. Astashkin, Nigel Kalton, and Fyodor A. Sukochev, Cesaro mean convergence of martingale differences in rearrangement invariant spaces, Positivity 12 (2008), no. 3, 387406, DOI 10.1007/s11117-007-2146-y. MR2421142(2010k:46015)

[4] T. Andô, On fundamental properties of a Banach space with a cone, Pacific J. Math. 12 (1962), 1163-1169. MR0150572(27 \#568)

[5] A. M. Bikchentaev, A block projection operator in normed ideal spaces of measurable operators (Russian, with English and Russian summaries), Izv. Vyssh. Uchebn. Zaved. Mat. 2 (2012), 86-91, DOI 10.3103/S1066369X12020107; English transl., Russian Math. (Iz. VUZ) 56 (2012), no. 2, 75-79. MR3076533

[6] Vladimir I. Chilin, Andrei V. Krygin, and Pheodor A. Sukochev, Extreme points of convex fully symmetric sets of measurable operators, Integral Equations Operator Theory 15 (1992), no. 2, 186-226, DOI 10.1007/BF01204237. MR1147280 (93g:46065) 
[7] V. I. Chilin and F. A. Sukochev, Weak convergence in non-commutative symmetric spaces, J. Operator Theory 31 (1994), no. 1, 35-65. MR1316983 (96e:46085)

[8] V. I. Chilin, A. A. Sedaev, and F. A. Sukochev, Weak compactness in Lorentz spaces, Uzb. Math. J. 1 (1993), 84-93 (in Russian).

[9] V. I. Chilin, P. G. Dodds, A. A. Sedaev, and F. A. Sukochev, Characterisations of KadecKlee properties in symmetric spaces of measurable functions, Trans. Amer. Math. Soc. 348 (1996), no. 12, 4895-4918, DOI 10.1090/S0002-9947-96-01782-5. MR1390973 (97d:46031)

[10] V. I. Chilin, P. G. Dodds, and F. A. Sukochev, The Kadec-Klee property in symmetric spaces of measurable operators, Israel J. Math. 97 (1997), 203-219, DOI 10.1007/BF02774037. MR 1441249 (98c:46129)

[11] Jacques Dixmier, von Neumann algebras, North-Holland Mathematical Library, vol. 27, North-Holland Publishing Co., Amsterdam-New York, 1981. With a preface by E. C. Lance; Translated from the second French edition by F. Jellett. MR641217 (83a:46004)

[12] Peter G. Dodds, Theresa K.-Y. Dodds, and Ben de Pagter, Noncommutative Banach function spaces, Math. Z. 201 (1989), no. 4, 583-597, DOI 10.1007/BF01215160. MR1004176 (90j:46054)

[13] Peter G. Dodds, Theresa K.-Y. Dodds, and Ben de Pagter, A general Markus inequality, Miniconference on Operators in Analysis (Sydney, 1989), Proc. Centre Math. Anal. Austral. Nat. Univ., vol. 24, Austral. Nat. Univ., Canberra, 1990, pp. 47-57. MR1060110 (91i:46072)

[14] Peter G. Dodds, Theresa K. Dodds, and Ben de Pagter, Weakly compact subsets of symmetric operator spaces, Math. Proc. Cambridge Philos. Soc. 110 (1991), no. 1, 169-182, DOI 10.1017/S0305004100070225. MR1104612(93e:46074)

[15] Peter G. Dodds, Theresa K.-Y. Dodds, and Ben de Pagter, Noncommutative Köthe duality, Trans. Amer. Math. Soc. 339 (1993), no. 2, 717-750, DOI 10.2307/2154295. MR.1113694 (94a:46093)

[16] P. G. Dodds, T. K. Dodds, F. A. Sukochev, and O. Ye. Tikhonov, A non-commutative YosidaHewitt theorem and convex sets of measurable operators closed locally in measure, Positivity 9 (2005), no. 3, 457-484, DOI 10.1007/s11117-005-1384-0. MR2188531 (2006h:46062)

[17] P. G. Dodds and D. H. Fremlin, Compact operators in Banach lattices, Israel J. Math. 34 (1979), no. 4, 287-320 (1980), DOI 10.1007/BF02760610. MR.570888 (81g:47037)

[18] P. G. Dodds and B. de Pagter, Completely positive compact operators on non-commutative symmetric spaces, Positivity 14 (2010), no. 4, 665-679, DOI 10.1007/s11117-010-0073-9. MR2741325(2011k:46088)

[19] P. G. Dodds and B. de Pagter, The non-commutative Yosida-Hewitt decomposition revisited, Trans. Amer. Math. Soc. 364 (2012), no. 12, 6425-6457, DOI 10.1090/S0002-9947-201205569-3. MR2958942

[20] P. G. Dodds and B. de Pagter, Normed Köthe spaces: a non-commutative viewpoint, Indag. Math. (N.S.) 25 (2014), no. 2, 206-249, DOI 10.1016/j.indag.2013.01.009. MR3151815

[21] P. G. Dodds, B. de Pagter, and F. Sukochev, Theory of Noncommutative Integration, unpublished monograph, to appear.

[22] P. G. Dodds, F. A. Sukochev, and G. Schlüchtermann, Weak compactness criteria in symmetric spaces of measurable operators, Math. Proc. Cambridge Philos. Soc. 131 (2001), no. 2, 363-384, DOI 10.1017/S0305004101005114. MR1857125 (2002f:46123)

[23] Thierry Fack and Hideki Kosaki, Generalized s-numbers of $\tau$-measurable operators, Pacific J. Math. 123 (1986), no. 2, 269-300. MR840845 (87h:46122)

[24] D. H. Fremlin, Stable subspaces of $L^{1}+L^{\infty}$, Proc. Cambridge Philos. Soc. 64 (1968), 625-643. MR0225154 (37 \#749)

[25] A. Grothendieck, Topological vector spaces, Translated from the French by Orlando Chaljub, Notes on Mathematics and its Applications, Gordon and Breach Science Publishers, New York-London-Paris, 1973. MR0372565 (51 \#8772)

[26] U. Haagerup, H. P. Rosenthal, and F. A. Sukochev, Banach embedding properties of non-commutative $L^{p}$-spaces, Mem. Amer. Math. Soc. 163 (2003), no. 776, vi+68, DOI 10.1090/memo/0776. MR 1963854 (2004f:46076)

[27] N. J. Kalton and F. A. Sukochev, Symmetric norms and spaces of operators, J. Reine Angew. Math. 621 (2008), 81-121, DOI 10.1515/CRELLE.2008.059. MR2431251 (2009i:46118)

[28] S. G. Krě̆n, Yu. İ. Petunīn, and E. M. Semënov, Interpolation of linear operators, Translations of Mathematical Monographs, vol. 54, American Mathematical Society, Providence, R.I., 1982. Translated from the Russian by J. Szücs. MR649411 (84j:46103) 
[29] A. V. Krygin, E. M. Sheremet'ev, and F. A. Sukochev, Conjugation of weak and measure convergence in noncommutative symmetric spaces, Dokl. Akad. Nauk UzSSR (1993), no. 2, 8-9 (in Russian).

[30] A. V. Krygin, E. M. Sheremet'ev, and F. A. Sukochev, Convergence in measure, weak convergence and structure of subspaces in symmetric spaces of measurable operators, unpublished manuscript, 1993.

[31] Peter Meyer-Nieberg, Banach lattices, Universitext, Springer-Verlag, Berlin, 1991. MR 1128093 (93f:46025)

[32] Edward Nelson, Notes on non-commutative integration, J. Functional Analysis 15 (1974), 103-116. MR0355628(50 \#8102)

[33] Erwin Neuhardt, Order properties of compact maps on $L^{p}$-spaces associated with von Neumann algebras, Math. Scand. 66 (1990), no. 1, 110-116. MR1060901 (91i:46076)

[34] B. de Pagter, H. Witvliet, and F. A. Sukochev, Double operator integrals, J. Funct. Anal. 192 (2002), no. 1, 52-111, DOI 10.1006/jfan.2001.3898. MR.1918492(2003g:46075)

[35] Timur Oikhberg and Eugeniu Spinu, Domination of operators in the non-commutative setting, Studia Math. 219 (2013), no. 1, 35-67, DOI 10.4064/sm219-1-3. MR.3139423

[36] Narcisse Randrianantoanina, Sequences in non-commutative $L^{p}$-spaces, J. Operator Theory 48 (2002), no. 2, 255-272. MR1938797 (2003h:46093)

[37] Yves Raynaud, On ultrapowers of non commutative $L_{p}$ spaces, J. Operator Theory 48 (2002), no. 1, 41-68. MR.1926043 (2003i:46069)

[38] Yves Raynaud and Quanhua Xu, On subspaces of non-commutative $L_{p}$-spaces, J. Funct. Anal. 203 (2003), no. 1, 149-196, DOI 10.1016/S0022-1236(03)00045-4. MR.1996870(2004h:46076)

[39] Kazuyuki Saitô, On the preduals of $W^{*}$-algebras, Tôhoku Math. J. (2) 19 (1967), 324-331. MR0226416 (37 \#2006)

[40] F. A. Sukochev and V. I. Chilin, A convergence criterion in regular noncommutative symmetric spaces (Russian, with Uzbek summary), Izv. Akad. Nauk UzSSR Ser. Fiz.-Mat. Nauk 4 (1990), 34-39, 90. MR.1108770 (92e:46129)

[41] F. A. Sukochev and V. I. Chilin, Symmetric spaces over semifinite von Neumann algebras (Russian), Dokl. Akad. Nauk SSSR 313 (1990), no. 4, 811-815; English transl., Soviet Math. Dokl. 42 (1991), no. 1, 97-101. MR1080637(92a:46075)

[42] Masamichi Takesaki, Theory of operator algebras. I, Springer-Verlag, New York-Heidelberg, 1979. MR.548728(81e:46038)

[43] M. Terp, $L^{p}$-spaces associated with von Neumann algebras, Notes, Copenhagen University (1981).

[44] E. V. Tokarev, Subspaces of certain symmetric spaces (Russian), Teor. Funkciǔ Funkcional. Anal. i Priložen. Vyp. 24 (1975), 156-161, iv. MR0626854 (58 \#30126)

[45] A. C. Zaanen, Riesz spaces. II, North-Holland Mathematical Library, vol. 30, North-Holland Publishing Co., Amsterdam, 1983. MR704021 (86b:46001)

School of Computer Science, Mathematics and Engineering, Flinders University, GPO Box 2100, Adelaide 5001, Australia

E-mail address: peter@csem.flinders.edu.au

Delft Institute of Applied Mathematics, Faculty EemCS, Delft University of TechNology, P.O. Box 5031, 2600 GA Delft, The Netherlands

E-mail address: b.depagter@tudelft.nl

School of Mathematics and Statistics, University of New South Wales, Kensington 2052, New South Wales, Australia

E-mail address: f.sukochev@unsw.edu.au 